\title{
Nulling interferometry: impact of exozodiacal clouds on the performance of future life-finding space missions
}

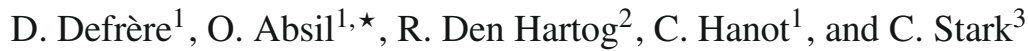

\author{
${ }^{1}$ Institut d'Astrophysique et de Géophysique, Université de Liège, 17 Allée du Six Août, 4000 Liège, Belgium \\ e-mail: defrere@astro.ulg.ac.be \\ 2 Netherlands Institute for Space Research, SRON, Sorbonnelaan 2, 3584 CA, Utrecht, The Netherlands \\ ${ }^{3}$ Department of Physics, University of Maryland, Box 197, 082 Regents Drive, College Park, MD 20742-4111, USA
}

Received 23 July 2009 / Accepted 5 October 2009

\begin{abstract}
Context. Earth-sized planets around nearby stars are being detected for the first time by ground-based radial velocity and space-based transit surveys. This milestone is opening the path toward the definition of instruments able to directly detect the light from these planets, with the identification of bio-signatures as one of the main objectives. In that respect, both the European Space Agency (ESA) and the National Aeronautics and Space Administration (NASA) have identified nulling interferometry as one of the most promising techniques. The ability to study distant planets will however depend on the amount of exozodiacal dust in the habitable zone of the target stars.

Aims. We assess the impact of exozodiacal clouds on the performance of an infrared nulling interferometer in the Emma X-array configuration. The first part of the study is dedicated to the effect of the disc brightness on the number of targets that can be surveyed and studied by spectroscopy during the mission lifetime. In the second part, we address the impact of asymmetric structures in the discs such as clumps and offset which can potentially mimic the planetary signal.

Methods. We use the DarwinSIM software which was designed and validated to study the performance of space-based nulling interferometers. The software has been adapted to handle images of exozodiacal discs and to compute the corresponding demodulated signal.

Results. For the nominal mission architecture with 2-m aperture telescopes, centrally symmetric exozodiacal dust discs about 100 times denser than the solar zodiacal cloud can be tolerated in order to survey at least 150 targets during the mission lifetime. Considering modeled resonant structures created by an Earth-like planet orbiting at 1 AU around a Sun-like star, we show that this tolerable dust density goes down to about 15 times the solar zodiacal density for face-on systems and decreases with the disc inclination.

Conclusions. Whereas the disc brightness only affects the integration time, the presence of clumps or offset is more problematic and can hamper the planet detection. Based on the worst-case scenario for debris disc structures, the upper limit on the tolerable exozodiacal dust density is approximately 15 times the density of the solar zodiacal cloud. This gives the typical sensitivity that we will need to reach on exozodiacal discs in order to prepare the scientific programme of future Earth-like planet characterisation missions.
\end{abstract}

Key words. instrumentation: high angular resolution - techniques: interferometric - circumstellar matter interplanetary medium - planetary systems - planetary systems: protoplanetary disks

\section{Introduction}

The possibility of identifying habitable worlds and even biosignatures from extrasolar planets currently contributes to the growing interest about their nature and properties. Since the first planet discovered around another solar-type star in 1995 (Mayor \& Queloz 1995), nearly 400 extrasolar planets have been detected and many more are expected to be unveiled by ongoing or future search programmes. Most extrasolar planets detected so far have been identified from the ground by indirect techniques, which rely on observable effects induced by the planet on its parent star. From the ground, radial velocity measurements are currently limited to the detection of planets about 2 times as massive as the Earth in orbits around Sun-like and low-mass stars (Mayor et al. 2009) while the transit method is limited to Neptune-sized planets (Gillon et al. 2007). Thanks to the very high precision photometry enabled by the stable space environment, the first space-based dedicated missions (namely CoRoT and Kepler) are

\footnotetext{
* FNRS Postdoctoral Researcher
}

now revealing Earth-sized extrasolar planets by transit measurements as well. Launched in 2006, CoRoT (Convection Rotation and planetary Transits) has detected its first extrasolar planets (e.g., Barge et al. 2008; Alonso et al. 2008; Léger et al. 2009) and is expected to unveil about 100 transiting planets down to a size of $2 R_{\oplus}$ around G0V stars and $1.1 R_{\oplus}$ around M0V stars over its entire lifetime for short orbital periods (Moutou et al. 2005). Launched in 2009, Kepler is currently extending the survey to Earth-sized planets located in the habitable zone of about $10^{5}$ main sequence stars (Borucki et al. 2007). Within 4 years, Kepler should have discovered several hundred of terrestrial planets with periods between one day and 400 days. After this initial reconnaissance by CoRoT and Kepler, the Space Interferometry Mission (SIM PlanetQuest) might provide unambiguously the mass of Earth-sized extrasolar planets orbiting in the habitable zone of nearby stars by precise astrometric measurements. With CoRoT and Kepler, we will have a large census of Earth-sized extrasolar planets and their occurrence rate as a function of various stellar properties. However, even though the composition 
of the upper atmosphere of transiting extrasolar planets can be probed in favorable cases (e.g., Richardson et al. 2007), none of these missions directly detects the photons emitted by the planets which are required to study the planet atmospheres and eventually reveal the signature of biological activity.

Detecting the light from an Earth-like extrasolar planet is very challenging due to the high contrast $\left(\sim 10^{7}\right.$ in the mid-IR, $\sim 10^{10}$ in the visible) and the small angular separation $(\sim 0.5 \mu \mathrm{rad}$ for an Earth-Sun system located at $10 \mathrm{pc}$ ) between the planet and its host star. A technique that has been proposed to overcome these difficulties is nulling interferometry (Bracewell 1978). The basic principle is to combine the beams coming from two telescopes in phase opposition so that a dark fringe appears on the line of sight, which strongly reduces the stellar emission. Considering the two-telescope interferometer initially proposed by Bracewell, the response on the plane of the sky is a series of sinusoidal fringes, with angular spacing of $\lambda / b$. By adjusting the baseline length $(b)$ and orientation, the transmission of the off-axis planetary companion can then be maximised. However, even when the stellar emission is sufficiently reduced, it is generally not possible to detect Earth-like planets with a static array configuration, because their emission is dominated by the thermal contribution of warm dust in our solar system as well as around the target stars (exozodiacal cloud). This is the reason why Bracewell proposed to rotate the interferometer so that the planetary signal is modulated by alternatively crossing high and low transmission regions, while the stellar signal and the background emission remain constant. The planetary signal can then be retrieved by synchronous demodulation. However, a rapid rotation of the array would be difficult to implement and the detection is highly vulnerable to low frequency drifts in the stray light, thermal emission, and detector gain. A number of interferometer configurations with more than two collectors have then been proposed to perform faster modulation and overcome this problem by using phase chopping (Angel \& Woolf 1997; Mennesson \& Mariotti 1997; Absil 2001). The principle of phase chopping is to synthetize two different transmission maps with the same telescope array, by applying different phase shifts in the beam combination process. By differencing two different transmission maps, it is possible to isolate the planetary signal from the contributions of the star, local zodiacal cloud, exozodiacal cloud, stray light, thermal, or detector gain. Phase chopping can be implemented in various ways (e.g. inherent and internal modulation, Absil 2006), and are now an essential part of future spacebased life-finding nulling interferometry missions such as ESA's DARWIN (Fridlund et al. 2006) and NASA's Terrestrial Planet Finder (TPF, Lawson et al. 2008). The purpose of this paper is to assess the impact of exozodiacal dust discs on the performance of these missions. After describing the nominal performance of DARWIN/TPF, the first part of the study is dedicated to centrally symmetric exozodiacal discs which are suppressed by phase chopping and only contribute through their shot noise. If they are too bright, exozodiacal discs can drive the integration time and we investigate the corresponding impact on the number of planets that can be surveyed during the mission lifetime. In the second part, we address the impact of asymmetric structures in the discs (such as clumps and offset) which are not canceled by phase chopping and can seriously hamper the planet detection process.

\section{Darwin/TPF overview}

Considerable effort have been expended in the past decade by both ESA and NASA to design a mission that provides the

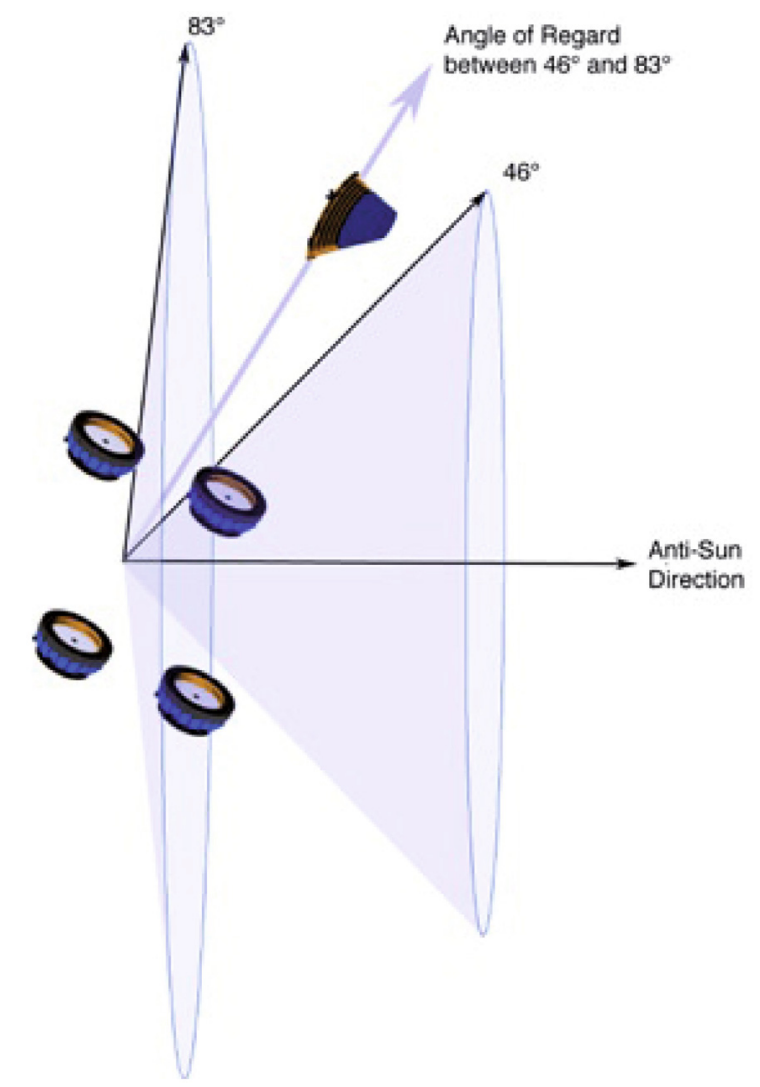

Fig. 1. Representation of the DARWIN/TPF space interferometer in its baseline "Emma X-array" configuration (Léger \& Herbst 2007). It includes 4 telescopes and a beam combiner spacecraft, deployed and observing at the Sun-Earth Lagrange point L2. At any given time, it can observe an annular region on the sky between $46^{\circ}$ and $83^{\circ}$ from the antisolar direction. During one Earth year, this annulus rotates and gives access to almost all regions of the celestial sphere.

required scientific performance while minimizing cost and technical risks. After the investigation of several interferometer architectures, these efforts culminated in 2005-2006 with two parallel assessment studies of the DARWIN mission, carried out by EADS Astrium and Alcatel-Alenia Space. Two array architectures have been thoroughly investigated during these industrial studies: the four-telescope $\mathrm{X}$-array and the Three-Telescope Nuller (TTN Karlsson et al. 2004). These studies included the launch requirements, payload spacecraft, and the ground segment during which the actual mission science would be executed. Almost simultaneously, NASA/JPL initiated a similar study for the Terrestrial Planet Finder Interferometer (TPF-I, Lawson et al. 2008). These efforts on both sides of the Atlantic have finally resulted in a convergence and consensus on mission architecture, the so-called non-coplanar or Emma-type X-array (represented in Fig. 1).

\subsection{Instrumental concept}

The baseline design consists of four 2-m aperture collector spacecraft, flying in rectangular formation and feeding light to the beam combiner spacecraft located approximately $1200 \mathrm{~m}$ above the array. This arrangement makes available baselines up to $70 \mathrm{~m}$ for nulling measurements and up to $400 \mathrm{~m}$ for the general astrophysics programme (constructive imaging). Note that the size of the collecting apertures has not yet been fixed and will influence the final cost of the mission. The optical layout 
Table 1. Instrumental parameters considered in this study for DARWIN/TPF.

\begin{tabular}{lc}
\hline \hline Instrumental parameters & Emma X-Array design \\
\hline Max. baselines [m] & $400 \times 67$ \\
Telescope diameter [m] & 2.0 \\
Field of regard & $46^{\circ}$ to $83^{\circ}$ \\
Optics temperature [K] & 40 \\
Detector temperature [K] & 8 \\
Quantum efficiency & $70 \%$ \\
Instrument throughput & \\
Science waveband $[\mu \mathrm{m}]$ & $10 \%$ \\
Modal filtering [ $\mu \mathrm{m}]^{b}$ & $6.0-20.0$ \\
Spectral resolution & 60 \\
Instrumental stability $^{c}$ & \\
$\quad$ - rms OPD error [nm] & $1.5 / 11.5-20.0$ \\
$\quad$ - rms amplitude error & $0.05 \%$ \\
\hline
\end{tabular}

${ }^{a}$ Excluding ideal beam combiner losses and coupling efficiency; ${ }^{b}$ see Sect. 3.2; ${ }^{c}$ see Sect. 3.4.

separates the nulling and imaging functions, the shortest baselines being used for nulling and the longest ones for imaging. This configuration has the advantage of allowing optimal tuning of the shorter dimension of the array for starlight suppression while keeping a significantly longer dimension to provide a rapid modulation of the planet signal as the array rotates. The X-array design is also appropriate to implement various techniques for removing instability noise, which is one of the dominant noise contributor (see Appendix A). The assessment studies settled on an imaging to nulling baseline ratio of $3: 1$, based on scientific and instrument design constraints. A larger ratio of $6: 1$ could nonetheless improve performance by simplifying noise reduction in the post-processing of science images (Lay 2006). The optical system architecture is represented by the block diagram in Fig. 2 with the following elements in the optical path:

- four spherical primary mirrors located on a virtual paraboloid and focusing the beam at the paraboloid's focal point. The virtual parabola focal length has been set to $1200 \mathrm{~m}$, resulting from a trade-off between differential polarisation effects and inter spacecraft metrology capability, as well as to enable the implementation of longer baselines for an imaging mode;

- transfer optics consist of all the equipment needed to collect and redirect the incoming beams towards fixed directions whatever the interferometer configuration. This includes mainly tip-tilt mirrors to handle array reconfiguration, a derotator to handle the array rotation and a common two or three mirror telescope to achieve beam collimation;

- optical delay lines (ODL) to adjust the optical path differences (OPD);

- fast steering mirrors to correct for tip-tilt errors;

- deformable mirrors to compensate for quasi-static errors such as defocus and astigmatism which are due to the use of spherical mirrors;

- achromatic $\pi$ phase shifters to produce the destructive interference of on-axis stellar light (using Fresnel Rhombs for instance, Mawet et al. 2007);

- dichroic beam splitters to separate the signals between the science waveband and the waveband used for metrology;

- a Modified Mach Zehnder (MMZ, Serabyn \& Colavita 2001) beam combiner;

- coupling optics to focus the outputs of the cross-combiner into single mode fibres. Chalcogenide fibres can cover

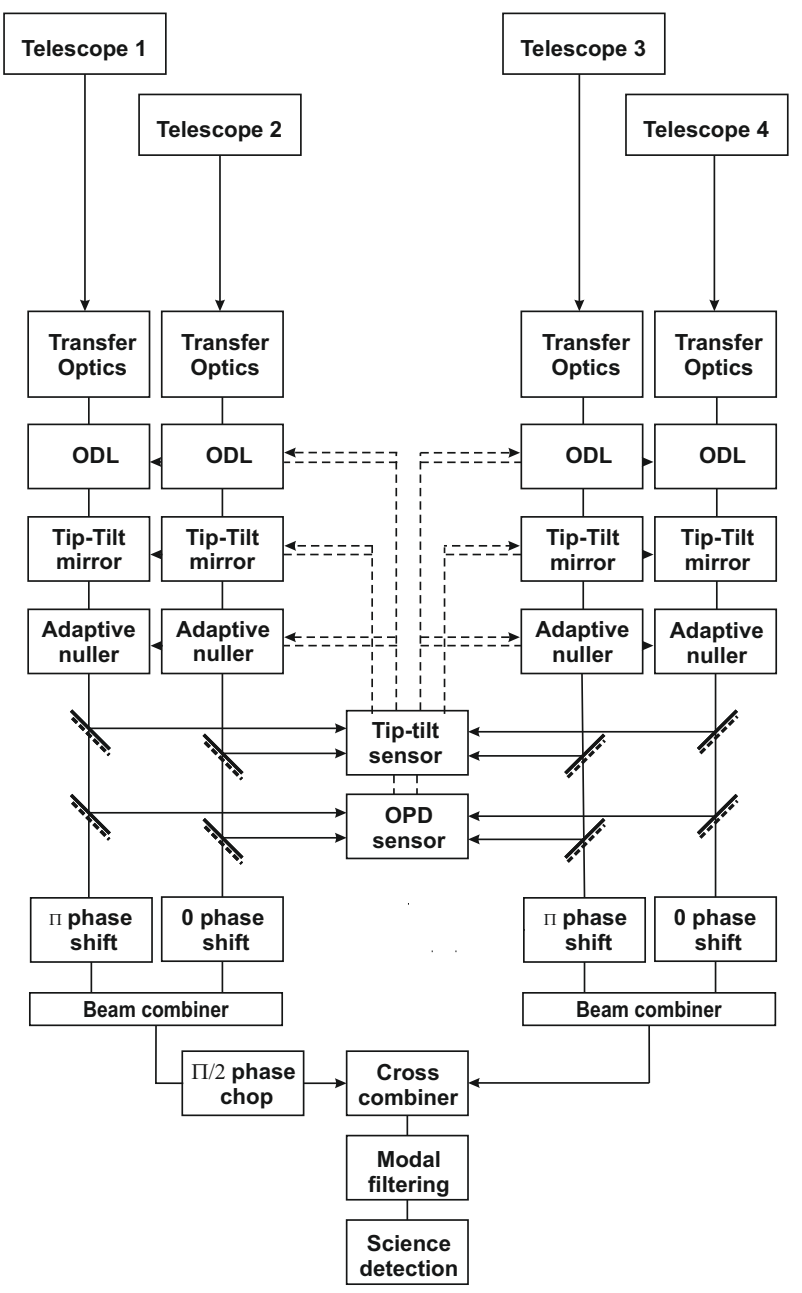

Fig. 2. Block diagram of the DARWIN/TPF optical layout. Feed-back signals driving the tip-tilt/OPD control are represented by dashed lines.

successfully the wavelength range 6.0-12 $\mu \mathrm{m}$ (Ksendzov et al. 2007) and silver halide fibres can be used for modal filtering in at least the $10.5-17.5 \mu \mathrm{m}$ spectral range (further investigations are however necessary to demonstrate that they are usable in the 17.5-20.0 $\mu \mathrm{m}$ wavelength range, Ksendzov et al. 2007);

- a detection assembly controlled at a temperature of $8 \mathrm{~K}$ and connected to the fibres.

DARWIN/TPF would be placed around the second Lagrange point (L2) by an Ariane 5 ECA vehicle. L2 is optimal to achieve passive cooling of the collector and beam combiner spacecraft down to $40 \mathrm{~K}$ by means of sunshades. An additional refrigerator within the beam combiner spacecraft cools the detector assembly to $8 \mathrm{~K}$. Due to the configuration of the array and the need for solar avoidance, the instantaneous sky access is limited to an annulus with inner and outer half-angles of $46^{\circ}$ and $83^{\circ}$ centred on the anti-sun vector (see Fig. 1, Carle 2005). This annulus transits the entire ecliptic circle during one year, giving access to almost the entire sky.

\subsection{Scientific objectives}

The main scientific objectives of DARWIN/TPF are the detection of rocky planets similar to Earth and the spectroscopic analysis of their atmospheres at mid-infrared wavelengths $(6-20 \mu \mathrm{m})$. In addition to presenting an advantageous star/planet contrast, 
Table 2. Parameters adopted for the performance simulations.

\begin{tabular}{ll}
\hline \hline Parameter & \\
\hline Configuration & Emma X-Array Design with a 6:1 aspect ratio (see Table 1) \\
Planet & Earth-sized planet with a constant temperature of 265 K across the habitable zone \\
Exozodiacal density & Three times our solar system, based on Kelsall model (Kelsall et al. 1998) \\
Mission duration & 5 years (2 years for survey and 3 years for characterisation) \\
Retargeting time & $6 \mathrm{~h}$ \\
Integration efficiency & $70 \%$ (accounting for overhead loss) \\
Target stars & Darwin All Sky Survey Catalogue (DASCC, Kaltenegger et al. 2008) \\
Earth-like planet per star $\left(\eta_{\oplus}\right)$ & 1 \\
Habitable zone & $0.7-1.5$ AU scaled with $L_{\star}^{1 / 2}$ \\
Time allocation & $10 \% \mathrm{~F}, 50 \% \mathrm{G}, 30 \% \mathrm{~K}$ and $10 \%$ M stars \\
Array rotation period & 50000 s (not scaled with the array baseline length) \\
$S N R$ threshold for detection & 5 \\
$S N R$ threshold for spectroscopy & $5\left(\mathrm{CO}_{2}, \mathrm{O}_{3}\right)$ and $10\left(\mathrm{CO}_{2}, \mathrm{O}_{3}, \mathrm{H}_{2} \mathrm{O}\right)$ \\
\hline
\end{tabular}

this wavelength range holds several spectral features relevant for the search of biological activity $\left(\mathrm{CO}_{2}, \mathrm{H}_{2} \mathrm{O}\right.$, and $\left.\mathrm{O}_{3}\right)$. The observing scenario of DARWIN/TPF consists of two phases, detection and spectral characterisation, whose relative duration can be adjusted to optimise the scientific return ${ }^{1}$. During the detection phase of the mission (nominally 2 years), DARWIN/TPF will examine nearby stars for evidence of terrestrial planets. A duration of 3 years is foreseen for the spectroscopy phase, for a total nominal mission lifetime of 5 years. An extension to 10 years is possible and will depend on the results obtained during the 5 first years. Such an extension could be valuable to observe more M stars (only $10 \%$ of the baseline time being attributed to them), search for big planets around a significantly larger sample of stars, and additional measurements on the most interesting targets already studied.

The DARWIN/TPF target star list has been generated from the Hipparcos catalogue, considering several criteria: the distance $(<30 \mathrm{pc})$, the brightness $(<12 V$-mag), the spectral type (F, G, $\mathrm{K}, \mathrm{M}$ main sequence stars), and the multiplicity (no companions within $1^{\prime \prime}$ ). The corresponding star catalogue contains 1229 single main sequence stars of which 107 are F, 235 are G, 536 are K, and 351 are $\mathrm{M}$ type (Kaltenegger et al. 2008). The survey of the DARWIN/TPF stars and the possible detection of terrestrial planets will start a new era of comparative planetology, especially by studying the relationship between habitability and stellar characteristics (e.g. spectral type, metallicity, age), planetary system characteristics (e.g. orbit), and atmospheric composition.

\section{Simulated performance}

\subsection{The science simulator}

The performance predictions presented in this paper have been computed using the DARWIN science SIMulator developed at ESA/ESTEC (DarwinSIM, den Hartog 2005b). This simulator has been subject to extensive validation the past few years and its performance predictions were recently reconciled with a similar mission simulator developed independently at NASA/JPL for the TPF mission (Lay et al. 2007). The two simulation tools have shown a very good agreement in SNR, giving similar integration times for all the Darwin/TPF targets with a discrepancy lower than $10 \%$ in average (Defrère et al. 2008b). These two simulators have the same basic purpose. For a given instrumental configuration and target catalogue, they assess the number of terrestrial planets that can be detected in the habitable zone of

\footnotetext{
1 The detection phase might not be necessary if the targets are identified in advance by radial velocity or astrometric surveys.
}

nearby main sequence stars and the number of possible followup spectroscopic observations during a nominal mission time. The duration of detection and spectroscopy phases can be adjusted to optimise the scientific return and is nominally set to 2 and 3 years respectively. The parameters and assumptions used are summarized in Table 2.

\subsubsection{Detection phase}

The starting point of the simulations is the target star catalogue. Given a specific interferometer architecture, the simulator first identifies the stars which are observable from L2. For each of these observable stars, the basic calculation consists of an assessment of the required integration times to achieve an userspecified SNR for broad-band detection of a hypothetical Earthlike planet located inside the habitable zone. The habitable zone is assumed to be located between 0.7 and $1.5 \mathrm{AU}$ for a G2V star and is scaled with the square root of the stellar luminosity $\left(L_{\star}^{1 / 2}\right)$. Since the location of the planet around the star is a priori unknown, the integration time is computed from the requirement that it should ensure the detection of a planet for at least $90 \%$ of the possible locations in the habitable zone. Assuming planets uniformly distributed along habitable orbits, this requires the computation of the probability distribution for finding a planet at a certain angular distance from the star. For each planetary position, the maximum SNR is computed by optimisation of the baseline length. The thermal flux of the habitable planet is assumed to be identical to that of Earth irrespective of the distance to the star. The exozodiacal clouds are simulated by assuming a nominal dust density 3 times larger than that in the solar system and a dust sublimation temperature of $1500 \mathrm{~K}$. Under the assumption that the exozodiacal emission is symmetric around the target star, it will be suppressed by phase chopping, and therefore only contributes to shot noise. The noise sources included are the shot noise contributions from stellar leakage, local and exozodiacal clouds, and instrumental infrared background. Instability noise is also present and is partly mitigated by phase chopping. A complete list can be found in Table 3.

After the initial integration time assessment for detection, the targets are sorted by ascending integration time, removing from the list the targets for which the total integration time exceeds the total time during which they are visible from L2. Considering a slew time for re-targeting (nominally $6 \mathrm{~h}$ ) and an efficiency for the remaining observing time of $70 \%$, the sorted list is cut off at the moment when the cumulative integration time exceeds the nominal survey period. Accounting for a specific time allocation for each spectral type (10\% F, 50\% G, 30\% K and 10\% M stars), 
Table 3. Detailed view of the various contributors to the noise budget, given in photo-electrons persecond over the 6-20 $\mu \mathrm{m}$ wavelength range for DARWIN/TPF in the Emma X-array configuration.

\begin{tabular}{lcccc}
\hline \hline & M0V $-05 \mathrm{pc}$ & $\mathrm{K} 0 \mathrm{~V}-10 \mathrm{pc}$ & $\mathrm{G} 2 \mathrm{~V}-15 \mathrm{pc}$ & $\mathrm{F} 0 \mathrm{~V}-20 \mathrm{pc}$ \\
\hline Optimum baseline [m] & 25 & 22 & 22 & 11 \\
Planetary modulation efficiency & 0.57 & 0.58 & 0.58 & 0.58 \\
Planetary coupling efficiency & 0.76 & 0.76 & 0.76 & 0.75 \\
\hline Stellar signal [e-/s] & $2.6 \times 10^{7}$ & $2.1 \times 10^{7}$ & $1.3 \times 10^{7}$ & $2.1 \times 10^{7}$ \\
Planetary signal [e-/s] & 5.52 & 1.39 & 0.62 & 0.35 \\
Photon noise [e-/s] & 0.459 & 0.388 & 0.358 & 0.365 \\
- Geometric leakage [e-/s] & 0.304 & 0.179 & 0.101 & 0.078 \\
- Null-floor leakage [e-/s] & 0.019 & 0.017 & 0.013 & 0.017 \\
- 3-zodi signal [e-/s] & 0.077 & 0.078 & 0.075 & 0.121 \\
- Local zodiacal signal [e-/s] & 0.260 & 0.260 & 0.260 & 0.260 \\
- Detected thermal [e-/s] & 0.101 & 0.101 & 0.101 & 0.101 \\
- Detected stray light [e-/s] & 0.166 & 0.166 & 0.166 & 0.166 \\
Dark current [e-/s] & 0.063 & 0.063 & 0.063 & 0.063 \\
Detector noise [e-/s] & 0.063 & 0.063 & 0.063 & 0.063 \\
Instability noise [e-/s] & 0.025 & 0.014 & 0.008 & 0.012 \\
- First order phase term [e-/s] & 0.021 & 0.008 & 0.002 & 0.001 \\
- Second order phase-amplitude [e-/s] & 0.014 & 0.012 & 0.007 & 0.012 \\
Total noise [e-/s] & 0.460 & 0.388 & 0.358 & 0.365 \\
Integrated $S$ NR (one rotation) & 16.0 & 4.4 & 2.1 & 1.1 \\
Integration time for $S N R=5$ [h] & 1.36 & 17.9 & 81.8 & 285 \\
\hline
\end{tabular}

The final SNRs are computed over a single rotation of $50000 \mathrm{~s}$ and for four targets representative of the target catalogue (see main text for further information).

the resulting list defines the number of targets that can be surveyed during the detection phase. The actual number of planets found will then depend on the number of terrestrial planets present in the habitable zone of target stars $\left(\eta_{\oplus}\right)$.

\subsubsection{Spectroscopy phase}

The number of targets which can be characterised by spectroscopy in a given time is computed similarly. The difference with the detection phase is that the integration times are computed for a given position in the habitable zone. The proper procedure would be to take into account all possible positions for the planet in a similar way to the detection phase but this would be far too time consuming. The strategy is then to consider only the most likely angular separation. Then, the total integration time is determined by the requirement to detect the absorption lines of $\mathrm{O}_{3}, \mathrm{CO}_{2}$ and $\mathrm{H}_{2} \mathrm{O}$ to a specified SNR. For the spectroscopy of $\mathrm{CO}_{2}$ and $\mathrm{O}_{3}$ (without $\mathrm{H}_{2} \mathrm{O}$ ), an $S N R$ of 5 would actually be sufficient for a secure detection (Fridlund 2005). Considering the spectroscopy of $\mathrm{H}_{2} \mathrm{O}$ is relatively more complex. Recent results suggest that, using a spectral resolution greater than 20 , an $S N R$ of 10 from 7.2 to $20 \mu \mathrm{m}$ would be sufficient for $\mathrm{H}_{2} \mathrm{O}$, $\mathrm{CO}_{2}$, and $\mathrm{O}_{3}$ spectroscopy (private communication with Selsis, Kaltenegger and Paillet). In particular, these results suggest that the $\mathrm{H}_{2} \mathrm{O}$ band located below $7.2 \mu \mathrm{m}$, which is much more timeconsuming than the $\mathrm{H}_{2} \mathrm{O}$ band beyond $17.2 \mu \mathrm{m}$, could be discarded.

Two types of spectroscopic analysis are considered: the staring spectroscopy, where the array is kept in a position such that the planet resides on a peak of the modulation map, and the rotating spectroscopy, where the array keeps on rotating with respect to the target system so that the planet moves in and out of the peaks of the modulation map. Staring spectroscopy is more efficient in terms of signal acquisition, but requires an accurate knowledge of the planetary orbit. As for the detection phase, the total spectroscopy integration time should not exceed the total time during which the target is visible during the characterisation phase. Accounting again for a given fraction of overhead loss, the targets are sorted with respect to ascending integration time, terminated where the cumulative time exceeds the length of the nominal characterisation period. The number of planets that can be characterised is then given with the assumption that there is one terrestrial planet in the habitable zone of each target star $\left(\eta_{\oplus}=1\right)$.

\subsection{Coupling efficiency}

Coupling the optical beams into optical fibers is an essential part of the wavefront correction process, which is required for deep nulling. The theoretical efficiency of light injection into an optical fiber depends on several parameters: the core radius of the fiber, its numerical aperture, the wavelength, the diameter of the telescope and its focal length (Ruilier \& Cassaing 2001). The method used in the simulator consists in choosing first the core radius of the fiber so as to ensure single-mode propagation over the whole wavelength range. The $f$-number of the coupling optics can then be optimised to give the maximum coupling efficiency at a chosen wavelength and more importantly, to provide a roughly uniformly high coupling efficiency across the whole wavelength band. The coverage of the full science band of DARWIN/TPF with one optical fiber is generally prohibited since the coupling efficiency drops rapidly with respect to the wavelength. Increasing the number of fibers improves the coupling efficiency but at the expense of complexity. In particular, it has been shown that the loss of targets for detection and spectroscopy is about $5 \%$ between the optimised 2-band and 3-band cases (den Hartog 2005a). Considering the use of two fibers (respectively on the $6.0-11.5 \mu \mathrm{m}$ and $11.5-20 \mu \mathrm{m}$ wavelength ranges), Fig. 3 shows the coupling efficiency for an on-axis source and for a source with a fixed off-axis angle, corresponding to an Earth orbit around a Sun at $10 \mathrm{pc}$. The coupling efficiency remains above $70 \%$ over the whole wavelength band of each fiber. 


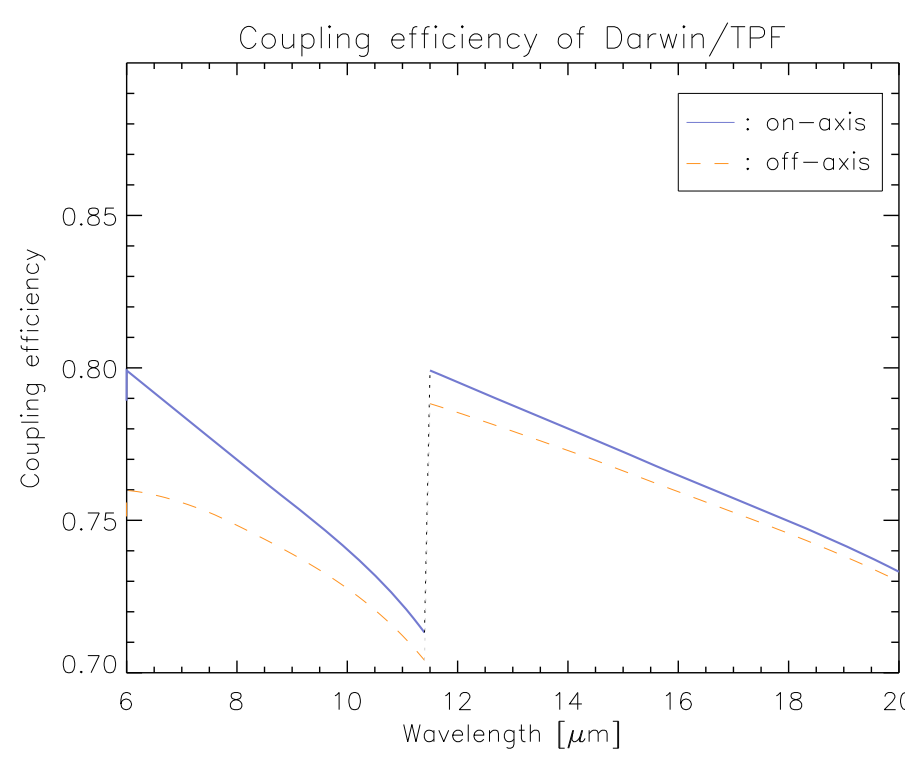

Fig. 3. Coupling efficiency for DARWIN/TPF with respect to the wavelength for an on-axis source and for a source with a fixed off-axis angle, corresponding to an Earth orbit around a Sun at $10 \mathrm{pc}$. The core radius is chosen so as to stay single-mode on the whole wavelength.

\subsection{Modulation efficiency}

The modulation of the planetary signal during the observation is a direct consequence of the chopping process which is mandatory to get rid of background noise sources such as exozodiacal and local zodiacal cloud emission. For the X-array configuration, the outputs of two Bracewell interferometers are combined with opposite phase shifts $( \pm \pi / 2)$ to produce two transmission maps (or "chop states"). Differencing the two transmission maps gives the chopped response of the interferometer, the so-called modulation map, which contains positive and negative values by construction (see Fig. 4). Since the value of the modulation map varies across the field-of-view, the position and flux of the planet cannot be unambiguously inferred and an additional level of modulation is mandatory. This is provided by the rotation of the interferometer (typically with a period of 1 day). The planetary signal is therefore modulated as shown on the right part of Fig. 4. In order to retrieve the planetary signal, the most common approach is correlation mapping, a technique closely related to the Fourier transform used for standard image synthesis (Lay 2005). The result is a correlation map, displayed for a single point source in the lower right part of Fig. 4. This represents the Point Spread Function (PSF) of the array. This process, illustrated here for a single wavelength, is repeated across the wavelength range, and the maps are co-added to obtain the net correlation map. The broad range of wavelengths planned for DARWIN/TPF greatly extends the spatial frequency coverage of the array, suppressing the side lobes of the PSF.

After chopping and rotation, the part of the incoming signal which is actually modulated and retrievable by synchronous demodulation is proportional to the "rotational modulation efficiency". It is shown for the X-array configuration in Fig. 5. It depends on the radial distance from the star and reaches a peak value of 0.56 with an asymptotic value of 0.44 . Since the planet position inside the habitable zone is a priori unknown, it is desirable that the effective modulation efficiency is as uniform as possible across the habitable zone to avoid too many reconfigurations of the interferometric array. Note that the rotational modulation efficiency for several array configurations has been investigated by Lay (2005).

\subsection{Instability noise}

Instability noise is defined as the component of the demodulated nulled signal that arises from phase, amplitude and polarisation errors (Lay 2004). The power spectra of these instrumental effects mix with each other so that perturbations at all frequencies, including DC, have an effect. Spacecraft vibrations, fringe tracking offset, control noise, longitudinal chromatic dispersion, and birefringence are at the origin of the phase errors whereas tip/tilt, defocus, beam shear, and differential transmission produce amplitude errors. These phase and amplitude errors induce a timedependent asymmetry between the two chop states so that the modulation map does not remain centered on the nominal position of the line of sight (i.e. the position of the star). Hence a fraction of the starlight survives the modulation process and mixes with the planet photons. Although a simple binary phase chop removes a number of these systematic errors, it has no effect on the dominant amplitude-phase cross terms and on the co-phasing errors. There is no phase chopping scheme that can remove the systematic errors without also removing the planetary signal. Three independent studies (Lay 2004; D'Arcio 2005; Chazelas et al. 2006) have reviewed the instrumental requirements on the DARWIN/TPF mission that reduce the instrumental stellar leakage to a sufficiently low level for Earth-like planet detection. Assuming the presence of $1 / \mathrm{f}$-type noise, these studies showed that the requirements on amplitude and phase control are not driven by the null-floor leakage, but by instability noise. Considering a Dual-Chopped Bracewell (DCB, Lay 2004) with 4-m aperture telescopes operating at $10 \mu \mathrm{m}$, the different analyses show that a null depth of $\sim 10^{-5}$ is generally sufficient to control the level of shot noise from the stellar leakage, but that a null depth of $\sim 10^{-6}$ is required to prevent instability noise from becoming the dominant source of noise ${ }^{2}$. In particular, a $10^{-6}$ null requires rms path control to within about $1.5 \mathrm{~nm}$, and rms amplitude control of about $0.1 \%$.

In order to relax these very stringent requirements, several techniques have been investigated (Lay 2005; Lane et al. 2006; Gabor et al. 2008). Discussion of these mitigation techniques is beyond the scope of this paper, where we assume that instability noise is sufficiently low to ensure the $\mathrm{H}_{2} \mathrm{O}$ spectroscopy at $7-\mu \mathrm{m}$ of an Earth-like planet orbiting around a Sun located at 15 pc. Applying the analytical method of (Lay 2004) to the Emma X-array with the parameters listed in Table 1, we derive the constraints on the instrument stability such that instability noise is dominated by shot noise by a factor 5 at $7 \mu \mathrm{m}$ over one rotation period $\left(t_{\text {rot }}\right)$ of $50000 \mathrm{~s}$ (with a spectral resolution of 20 ). Considering $1 / \mathrm{f}$-type PSDs defined on the $\left[1 / t_{\text {rot }}, 10^{4}\right] \mathrm{Hz}$ range, this corresponds to residuals rms OPD and amplitude errors of about $1.5 \mathrm{~nm}$ and $0.05 \%$ respectively. These values will be used thorough this study (see appendix A for further details). Although our computation has been done at $7 \mu \mathrm{m}$ where instability noise is much higher than at $10 \mu \mathrm{m}$, these constraints are not far from the values derived by Lay (2004) for two reasons. First, the telescopes considered here are smaller so that shot noise is relatively more dominant than in the previous analyses (shot noise is proportional to the square root of the stellar flux while the planetary signal and instability noise are directly

2 The current state-of-the-art for broadband nulling experiments is a $10^{-5}$ null which has been recently demonstrated at $10 \mu \mathrm{m}$ $(\Delta \lambda /$ lambda $=34 \%)$ with the adaptive nuller (Peters et al. 2009, in prep.). 


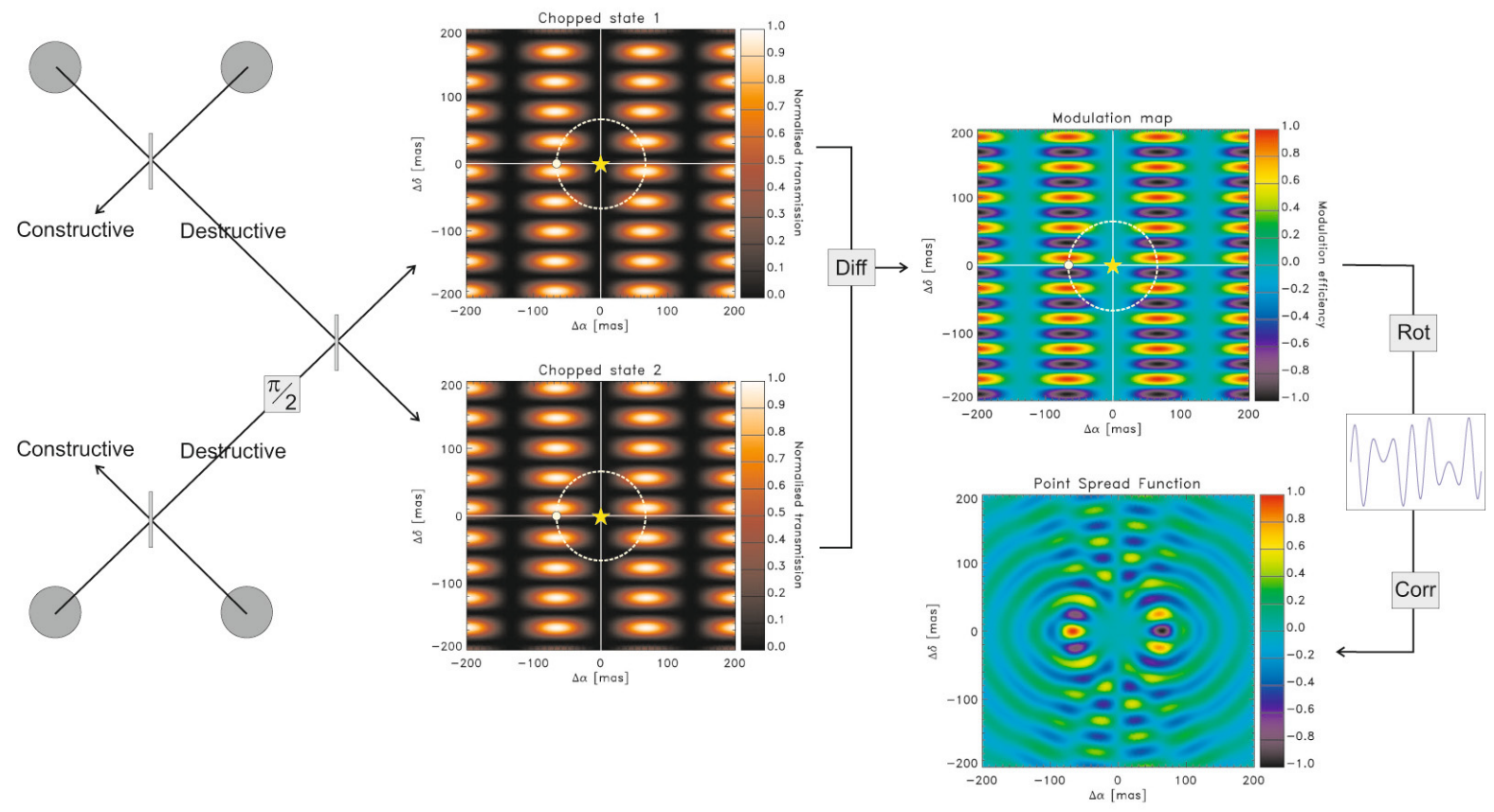

Fig. 4. Overview of phase chopping for the $\mathrm{X}$-array configuration. Combining the beams with different phases produces two conjugated transmission maps (or chop states), which are used to produce the chopped response. Array rotation then locates the planet by cross-correlation of the modulated chopped signal with a template function.

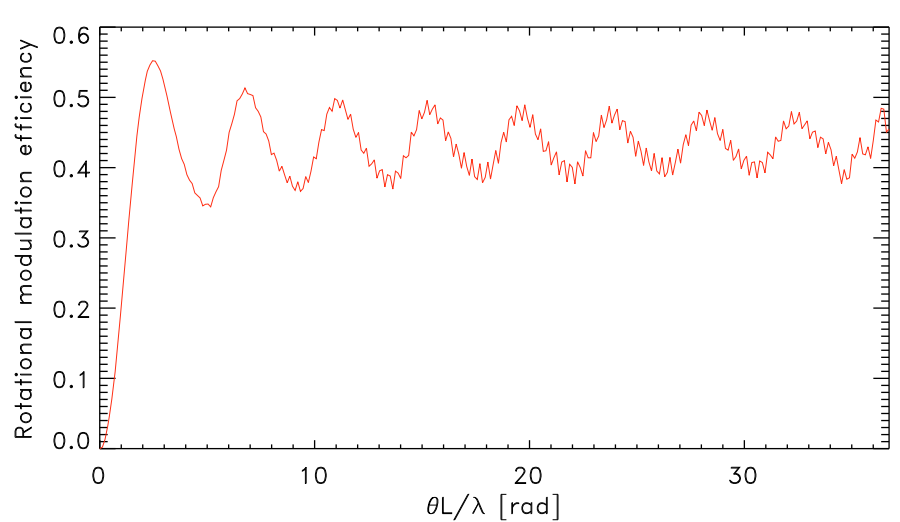

Fig. 5. Rotational modulation efficiency for the Emma X-array with a 6:1 aspect ratio.

proportional to the stellar flux). Secondly, the interferometer configuration is stretched by comparison with the DCB so that the planetary signal is modulated at higher frequencies, where the instability noise is lower assuming 1/f-type PSDs.

\subsection{Signal-to-noise analysis}

In this section, we present the different sources of noise simulated by DarwinSIM and the level at which they contribute to the final SNR for four targets representative of the DARWIN/TPF catalogue: an M0V star located at $5 \mathrm{pc}$, a K0V star at $10 \mathrm{pc}$, a $\mathrm{G} 2 \mathrm{~V}$ star at $15 \mathrm{pc}$ and an F0V star at $20 \mathrm{pc}$. The noise budget of each source is shown in Table 3 for a single rotation of $50000 \mathrm{~s}$ and for the optimum baseline length (computed by minimizing the integration time). The different contributors are described hereafter.

- The stellar signal represents the total number of photoelectrons that are generated by stellar photons detected in both constructive and destructive outputs.
- The planetary signal is the demodulated amount of photoelectrons that come from an Earth-like planet located at $1 \mathrm{AU}$ from the star.

- Shot noise is due to the statistical arrival process of the photons from all sources. It comes from the contributions from stellar leakage, the exozodiacal dust, the local zodiacal cloud, the thermal emission from the telescopes, and the stray light.

- Geometric stellar leakage accounts for the imperfect rejection of the stellar photons due to the finite size of the star and the non-null response of the interferometer for small offaxis angles.

- Null-floor leakage accounts for the stellar photons that leak through the output of the interferometer due to the influence of instrumental imperfections such as co-phasing errors, wavefront errors or mismatches in the intensities of the beams.

- The 3-zodi signal is the shot noise contribution from the circumstellar disc, assumed to be face-on and to follow the same model as in the solar system (Kelsall et al. 1998), except for a global density factor of 3 .

- Local zodiacal signal is the shot noise contribution from the solar zodiacal cloud, taking into account the spacecraft location at L2 and the pointing direction.

- Thermal background accounts for the emission of the telescopes.

- Stray light is made of the photons originating from outside the interferometer and which do not follow the nominal route to the detector. It includes scattered light from the target star, thermal photons from the instrument and any solar photon that are scattered into the instrument. We assume a nominal value of 10 photons per second and per spectral channel.

- Dark current is the constant response produced by the detector when it is not actively being exposed to light. We consider a nominal value of of 4 electrons rms per read and per spectral channel. 

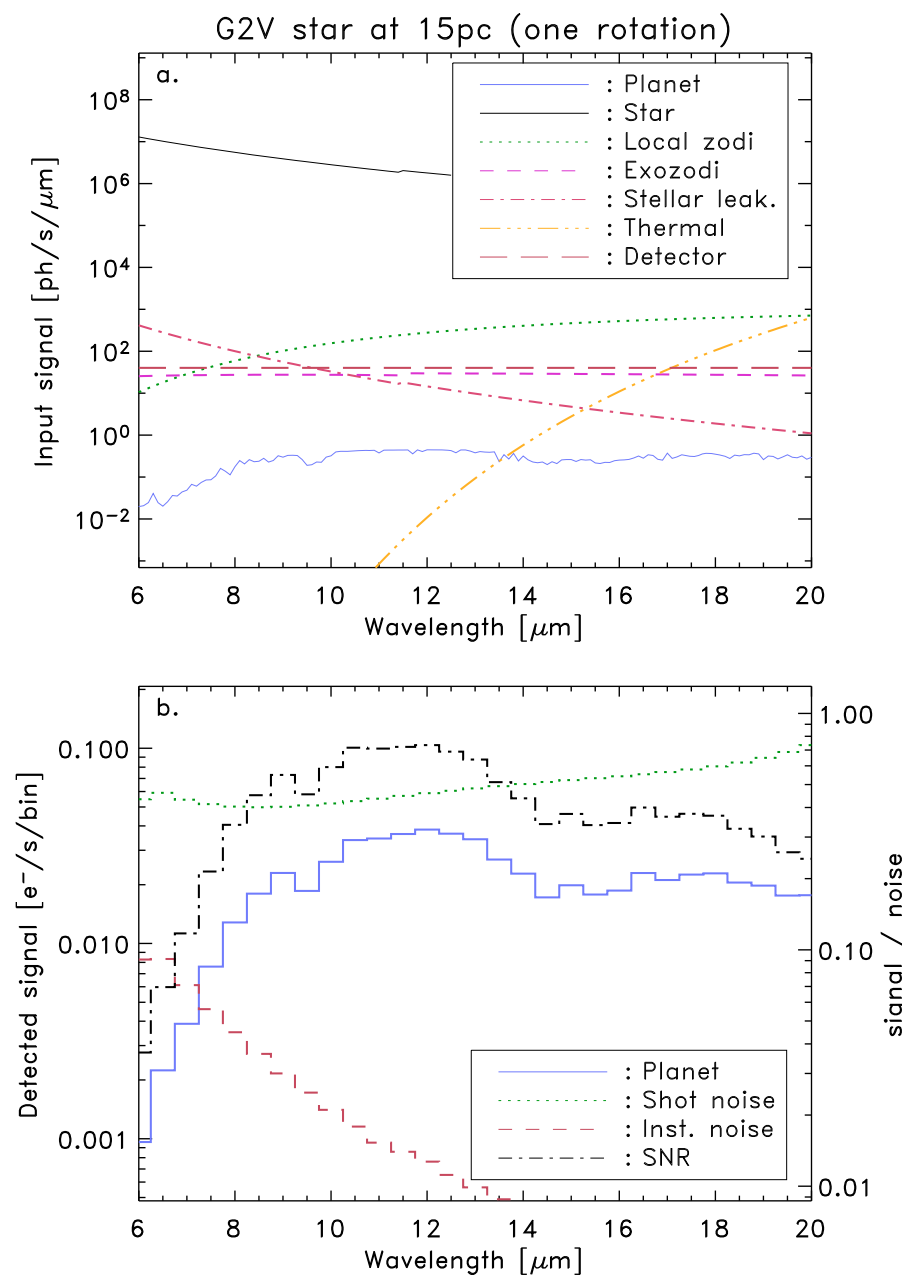

Fig. 6. Input a) and detected b) signals for an Earth-like planet orbiting at $1 \mathrm{AU}$ around a G2V star located at $15 \mathrm{pc}$. The demodulated signals are computed over a single rotation of $50000 \mathrm{~s}$.

- Detector noise is computed assuming a read-out noise of 4 electrons rms and a typical read-out frequency of $1 \mathrm{~Hz}$.

- Instability noise has been discussed in Sect. 3.4. It is computed for rms OPD and amplitude errors of $1.5 \mathrm{~nm}$ and $0.05 \%$ respectively (and defined on $1 / \mathrm{f}$-type power spectra).

As expected, shot noise is the dominant contributor for all targets (we have derived the constraints on the instrument stability so that instability noise is not dominant in Sect. 3.4). The emission of the local zodiacal cloud is generally the dominant source of shot noise but geometric stellar leakage and the exozodiacal dust also produce a significant part of it. Instability noise is dominated by second order phase-amplitude cross terms as pointed out by Lay (2004) and contributes weakly to the integrated SNR. The dependence on the wavelength is shown in Fig. 6 for the $\mathrm{G} 2 \mathrm{~V}$ star located at $15 \mathrm{pc}$. The upper figure represents the different input signals, showing that stellar leakage dominates at short wavelengths while the local zodiacal cloud emission is dominant at long wavelengths. The lower figure represents shot noise, instability noise and the detected signal from the planet. The SNR is also represented and measured on the right-hand side vertical axis. It is maximum around $10 \mu \mathrm{m}$, where the planetary signal peaks, and decreases rapidly toward short wavelengths, suggesting that the spectroscopy at these wavelengths would be the most time-consuming. Integrating the signal and noise sources from 6 to $20 \mu \mathrm{m}$ gives an $S N R$ of about 2 (see Table 3 ) so that about
Table 4. Expected performance in terms of number of stars surveyed and planets characterised during the nominal 5-year mission for various telescope diameters and planet radii.

\begin{tabular}{lccc|cc}
\hline \hline Telescope diameter & $1-\mathrm{m}$ & $2-\mathrm{m}$ & $4-\mathrm{m}$ & $2-\mathrm{m}$ & $2-\mathrm{m}$ \\
Planet radius $\left[R_{\oplus}\right]$ & 1 & 1 & 1 & 1.5 & 2 \\
\hline Detection & & & & & \\
Surveyed (5 years) & 89 & 303 & 813 & 590 & 921 \\
Surveyed (2 years) & 58 & 189 & 497 & 370 & 564 \\
\# F stars & 3 & 10 & 35 & 27 & 46 \\
\# G stars & 11 & 43 & 136 & 96 & 164 \\
\# K stars & 14 & 61 & 183 & 130 & 206 \\
\# M stars & 30 & 75 & 143 & 117 & 148 \\
\hline Spectroscopy & & & & & \\
Staring $\left(\mathrm{CO}_{2}, \mathrm{O}_{3}\right)$ & 20 & 64 & 199 & 132 & 234 \\
Rotating $\left(\mathrm{CO}_{2}, \mathrm{O}_{3}\right)$ & 20 & 43 & 127 & 89 & 159 \\
\# F stars & 0 & 2 & 5 & 4 & 8 \\
\# G stars & 2 & 7 & 25 & 17 & 36 \\
\# K stars & 2 & 10 & 40 & 24 & 46 \\
\# M stars & 16 & 24 & 67 & 44 & 69 \\
Staring ( $\left.\mathrm{H}_{2} \mathrm{O}\right)$ & 15 & 32 & 101 & 71 & 121 \\
Rotating $\left(\mathrm{H}_{2} \mathrm{O}\right)$ & 11 & 21 & 60 & 48 & 83 \\
\# F stars & 0 & 1 & 2 & 2 & 4 \\
\# G stars & 0 & 4 & 11 & 8 & 16 \\
\# K stars & 2 & 5 & 18 & 11 & 22 \\
\# M stars & 9 & 11 & 39 & 27 & 41 \\
\hline
\end{tabular}

All stars are assumed to host a planet in the habitable zone and to be surrounded by an exozodiacal cloud 3 times denser that in the solar system.

6 rotations of the interferometric array would be necessary to achieve the detection of the planet with an $S N R$ of 5. For spectroscopy, the integration time has to be much longer since the individual SNR in each spectral channel is significantly lower. For instance, about 150 rotations of the interferometric array would be necessary to achieve an $S N R$ of 10 at $10 \mu \mathrm{m}$.

\subsection{Expected performance}

Considering the assumptions given in Table 2, the simulated performance of DARWIN/TPF is shown in Table 4 for various aperture sizes and planet radii. Considering Earth-radius planets within the habitable zone, about 200 stars, well spread among the four selected spectral types, can be surveyed during the nominal 2-year detection phase. This number reaches about 500 with 4-m aperture telescopes. DARWIN/TPF will thus provide statistically meaningful results on nearby planetary systems. As already indicated by Table 3, nearby $\mathrm{K}$ and $\mathrm{M}$ dwarfs are the best-suited targets in terms of Earth-like planet detection capabilities.

For the spectroscopy phase, a required $S N R$ of 5 has been assumed for the detection of $\mathrm{CO}_{2}$ and $\mathrm{O}_{3}$, as discussed in Sect. 3.1. For the full characterisation (i.e. searching for the presence of $\mathrm{H}_{2} \mathrm{O}, \mathrm{CO}_{2}$, and $\mathrm{O}_{3}$ ), the required $S N R$ has been fixed to 10 on the $7.2-20-\mu \mathrm{m}$ wavelength range. With these assumptions, $\mathrm{CO}_{2}$ and $\mathrm{O}_{3}$ could be searched for about 40 planets (resp. 60) with rotational spectroscopy (resp. staring spectroscopy) while $\mathrm{H}_{2} \mathrm{O}$ could potentially be detected on 20 (resp. 30) planets during the 3 -year characterisation phase. These values would be roughly halved for 1-m aperture telescopes. Although staring spectroscopy presents (as expected) better results, rotational spectroscopy is more secure since it does not rely on an accurate localisation of the planet. It is also interesting to note that in the case of planets with radii 1.5 time as large as that of Earth, 
D. Defrère et al.: Impact of exozodiacal clouds on the performance of future life-finding space missions

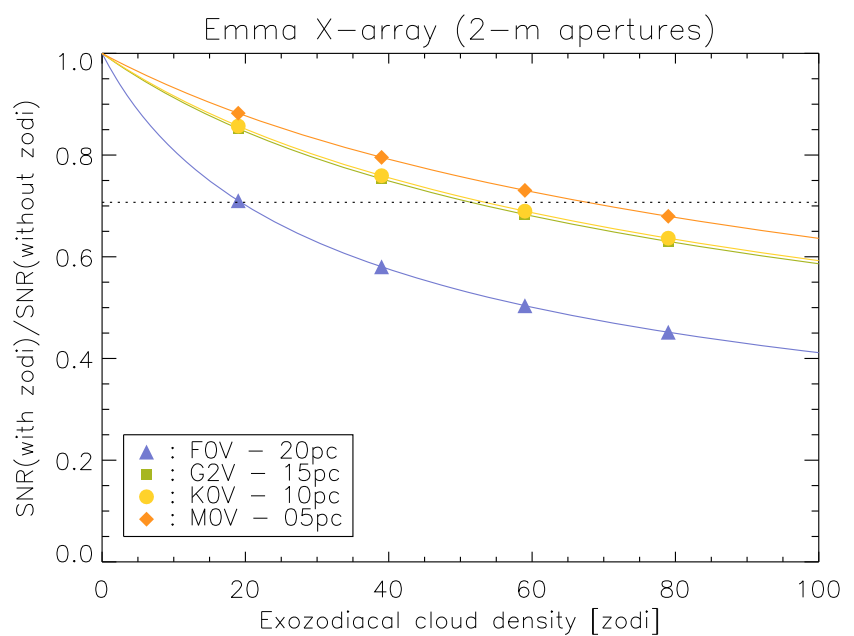

Fig. 7. Impact of the exozodiacal dust density on the SNR for different target stars. The exozodiacal disc is assumed to follow the Kelsall model (Kelsall et al. 1998) and to be seen in face-on orientation. The horizontal dotted line corresponds to an increase of integration time by a factor 2 with respect to the 0 -zodi case.

the number of planets for which $\mathrm{H}_{2} \mathrm{O}$ spectroscopy could be performed is doubled.

\section{Impact of the exozodiacal cloud density}

The amount of exozodiacal dust in the habitable zone of nearby main sequence stars is one of the main design drivers for the DARWIN/TPF mission. Depending on their morphology and brightness, exozodiacal dust clouds can seriously hamper the capability of a nulling interferometer to detect and characterise habitable terrestrial planets. Under the assumption that it is centrally symmetric around the target star, the exozodiacal cloud is suppressed by the chopping process, and therefore only contributes to shot noise. An exozodiacal cloud similar to the local zodiacal disc emits 350 times as much flux at $10 \mu \mathrm{m}$ than an Earth-like planet, so that it generally drives the integration time as the disc becomes a few times denser than the local zodiacal cloud. A previous study performed for the DCB with 3-m aperture telescopes observing a G2V star located at $10 \mathrm{pc}$ has led to the conclusion that detecting Earth-like planets around a star for which the exozodiacal cloud density is larger than 20 zodis would be difficult (Beichman et al. 2006). Nevertheless, the tolerable amount of dust around a nearby main sequence star highly depends on several parameters such as the telescope size, the target distance and spectral type. Another parameter which can affect the performance of the interferometer is the presence of asymmetric structures in the exozodiacal disc such as clumps or offset due to the presence of planets. These asymmetries have a different impact on the mission performance because they introduce a signal which is not perfectly suppressed by phase chopping and can mimic the planetary signal. This section is focused on the impact of the exozodiacal dust density on the integration time and the consequence on the number of targets that can be surveyed during the mission lifetime. The impact of asymmetric structures is discussed in Sect. 5.

\subsection{Analysis per individual target}

Currently, very little is known about the amount of exozodiacal dust in the habitable zone of nearby main sequence stars. First results have been obtained only very recently using classical infrared interferometry at the CHARA array (Mount Wilson,

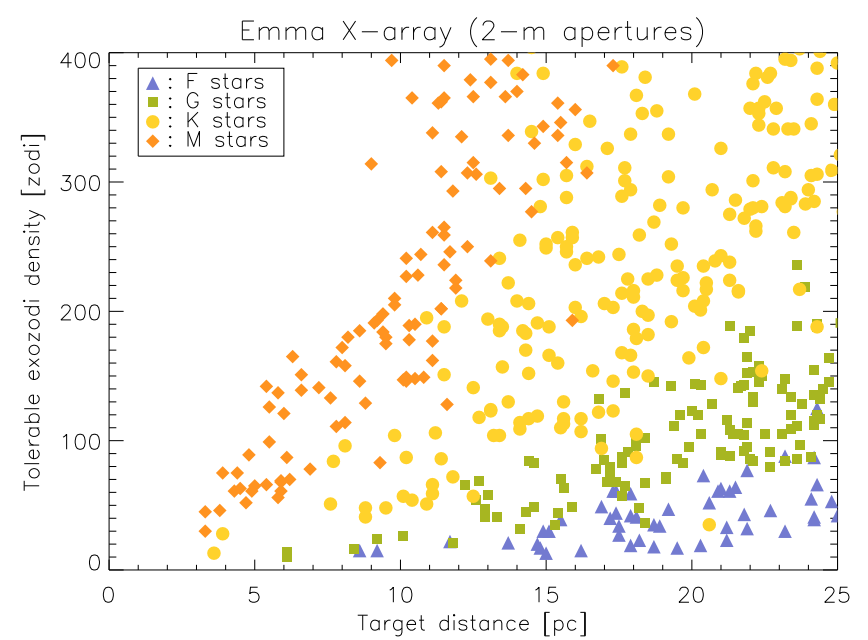

Fig. 8. Maximum number of zodis with respect to the target distance for the DARWIN/TPF target stars. The maximum number of zodis corresponds to an increase of integration time by a factor two with respect to the 0 -zodi case.

USA) and at the VLTI (Cerro Paranal, Chile). These instruments have revealed the presence of hot dust in the inner part of planetary systems around a few nearby main sequence stars with a sensitivity of approximately one thousand zodis (Absil et al. 2006; Di Folco et al. 2007; Absil et al. 2008b, 2009; Akeson et al. 2009). Recent observations using ground-based nulling interferometry at the Keck observatory (Mauna Kea, USA) have shown improved sensitivity to exozodiacal dust clouds of a few hundred zodis (Stark et al. 2009). Given the lack of information on exozodiacal clouds with densities of a few zodis, we investigate in this section the impact of exozodiacal dust density on the performance of DARWIN/TPF.

Considering centrally symmetric face-on exozodiacal discs, Fig. 7 shows the SNR (normalised to the SNR for no exozodiacal cloud) integrated over the 6-20 $\mu$ m wavelength range as a function of the exozodiacal dust density for the 4 typical target stars used in Sect. 3.5. We consider the normalised SNR because it does not depend on the integration time (the planetary signal is removed from the equation) which has the advantage to provide a common basis to compare the different target stars. Looking at Fig. 7, the impact of the exozodiacal dust density on the normalised SNR is particularly harmful for the hottest target stars which present the brightest exozodiacal discs. For the F0V star located at $20 \mathrm{pc}$, the normalised SNR is reduced by a factor of about 2.5 between the 0 and 100-zodi cases while for the M0V star located at $5 \mathrm{pc}$ it is only reduced by a factor of about 1.5. Assuming that the integration time should not be twice longer than in the 0-zodi case (see the horizontal dotted curve), the maximum number of zodis are about 70, 50, 50 and 20 respectively for the M0V star located at $5 \mathrm{pc}$, the K0V star at $10 \mathrm{pc}$, the G2V star at $15 \mathrm{pc}$ and the F0V star at $20 \mathrm{pc}$.

The distance of the star also plays an important role. As the distance to the target system increases, the flux collected from the exozodiacal cloud decreases while the flux collected from the local zodiacal cloud remains the same for all targets. The contribution of the exozodiacal dust cloud to the noise level becomes therefore relatively less important so that a higher dust density can be tolerated around the target. This explains why the curves of the G2V star and the K0V star almost coincide despite the fact that the G2V star is hotter. This behavior is illustrated in Fig. 8, showing the maximum number of zodis with respect to 


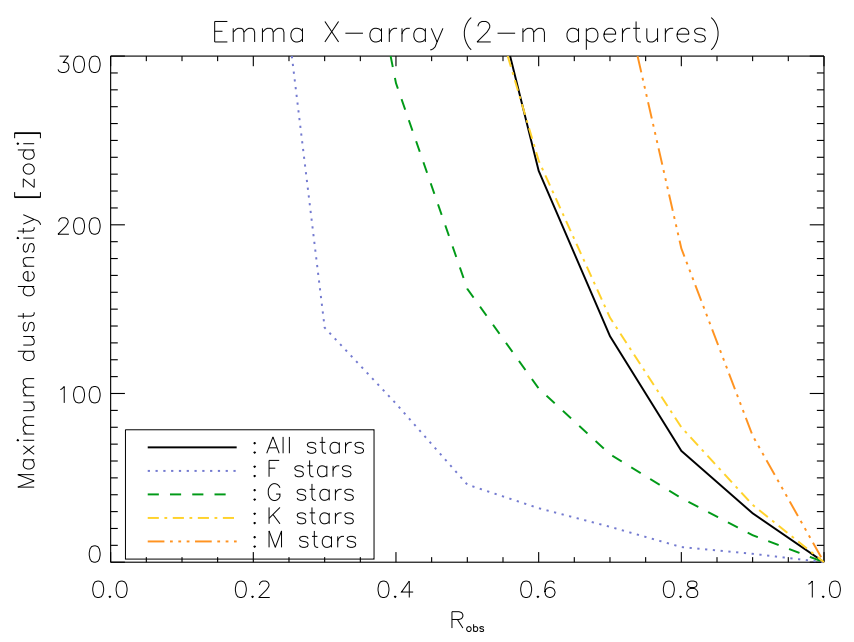

Fig. 9. Tolerable exozodiacal dust density as a function of the number of targets that can be observed during the mission lifetime normalised to the zodi-free case ( $\left.R_{\text {obs }}\right)$, accounting for overheads and a specific time allocation for each spectral type (10\% F, 50\% G, 30\% K and 10\% M).

the target distance for the whole DARWIN/TPF catalogue. This maximum number of zodis corresponds to an increase of integration time by a factor two with respect to the 0 -zodi case. It depends on the target distance and spectral type, and can take a value from few zodis up to several hundred zodis for the most distant stars. For a given distance to the target system, the maximum number of zodis increases with the stellar temperature, the zodi constraint being more severe on $F$ stars than on $M$ stars, while for a given spectral type, the zodi tolerance increases with the target distance.

\subsection{Tolerable dust density}

So far we have examined the maximum exozodiacal dust density for each target which corresponds to an integration time per target equals to twice the zero-zodi integration time. However, this does not tell us anything about the total number of stars that could be observed over the survey time of the mission. Here we derive the exozodiacal dust density that can be tolerated around nearby main sequence stars so that a given number of stars can be observed during the nominal mission lifetime.

To calculate the total number of targets that can be surveyed during the mission lifetime, we compute the integration time for each observable target as a function of the exozodiacal dust density and add them in ascending order as described in Sect. 3.1. Considering a slew time of $6 \mathrm{~h}$ and an efficiency for the remaining observing time of $70 \%$, the list is cut off when the cumulative integration time exceeds the nominal survey period. Applying this procedure to each spectral type (with the corresponding time allocation, see Table 2) and to the whole target list, Fig. 9 shows the tolerable dust density as a function of $R_{\mathrm{obs}}$, the ratio of the number of targets that can be observed during the mission lifetime in the presence of exozodiacal clouds of the given density to the number of targets that can be observed during the mission lifetime in the absence of exozodiacal clouds. The corresponding number of target stars that can be observed can easily be computed using Table 4.

As exozodiacal discs become denser, $R_{\text {obs }}$ decreases so that the tolerable dust density depends on the goal of the mission in terms of the number of stars that have to be observed. In order to survey approximately $50 \%$ of the stars that could be observed

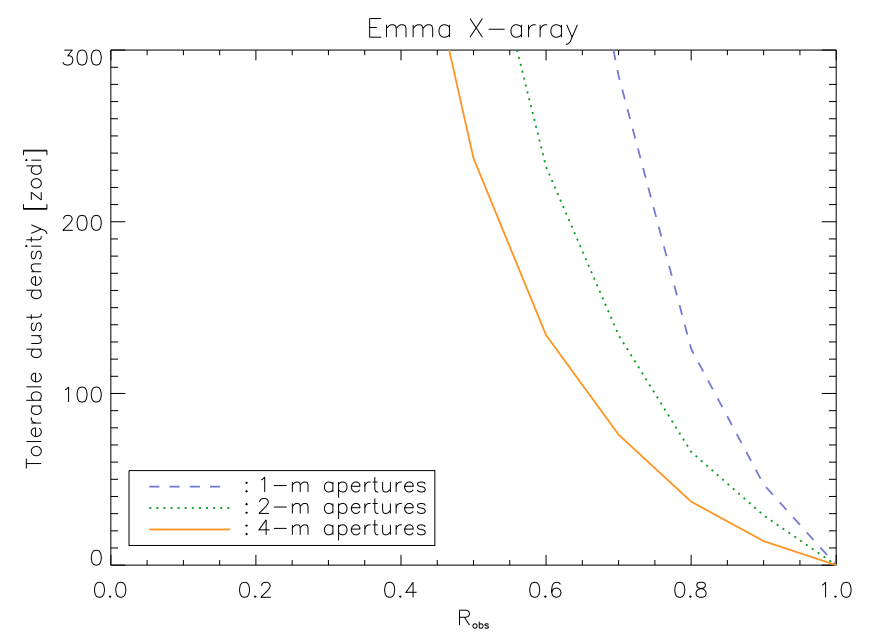

Fig. 10. Tolerable exozodiacal dust density for different aperture sizes as a function of the number of targets that can be observed during the mission lifetime normalised to the zodi-free case $\left(R_{\mathrm{obs}}\right)$. The results are computed for the nominal mission lifetime accounting for overheads and a specific time allocation for each spectral type $(10 \% \mathrm{~F}, 50 \% \mathrm{G}$, $30 \% \mathrm{~K}$ and $10 \% \mathrm{M}$ ).

in the absence of exozodiacal discs, a dust density as high as 400 zodis can be tolerated, while only 30 zodis are tolerable to observe $90 \%$ of the stars. Considering that at least 150 targets $^{3}$ have to be observed during the mission lifetime (about $75 \%$ of the stars), exozodiacal discs with a density of about 100 zodis can be tolerated around the targets stars. This tolerable dust density is computed for the whole catalogue assuming that the time allocation on each spectral type is maintained. In practice, the effect of exozodiacal dust is more pronounced for early type stars. This behavior is illustrated in Fig. 9 for each spectral type. For a dust density of 100 zodis, about 40\%, 60\%, 80\% and $90 \%$ of the F, G, K and M stars respectively can still be surveyed. Conversely, in order to survey at least $75 \%$ of the stars, the tolerable dust densities are about 10, 50, 100 and 300 zodis for F, $\mathrm{G}, \mathrm{K}$ and $\mathrm{M}$ stars respectively.

These results show how important it is to observe in advance the DARWIN/TPF targets in order to maximize the number of stars that can be surveyed during the mission lifetime. Ground-based nulling instruments like LBTI ("Large Binocular Telescope Interferometer", Hinz et al. 2008) and ALADDIN ("Antarctic L-band Astrophysics Discovery Demonstrator for Interferometric Nulling", Absil et al. 2008a) would be ideal to reach the detection of 50-zodi exozodiacal discs with a sky coverage sufficient to observe almost the entire DARWIN/TPF catalogue.

\subsection{Influence of the telescope size}

Increasing the telescope diameter has different influences on the individual signal and noise sources. The planetary signal increases as $\mathrm{D}^{2}$ while the shot noise contributions from geometric leakage and exozodiacal cloud increase as $\mathrm{D}$. The relative contribution from the local zodiacal cloud to shot noise is reduced for larger aperture telescopes, due to the smaller field-ofview. Since the local zodiacal cloud emission is generally one of

\footnotetext{
3 Surveying the habitable zone of at least 150 targets with $90 \%$ completeness has been defined by both ESA and NASA as the minimum mission requirement for DARWIN/TPF-I.
} 
the dominant noise sources, the relative impact of the exozodiacal cloud density on the SNR becomes therefore more significant for larger telescopes. Using the assumptions of Table 2, this behavior is illustrated in Fig. 10, which shows the tolerable exozodiacal dust density with respect to $R_{\mathrm{obs}}$ for different aperture sizes.

For a given dust density, the loss of observable targets during the mission lifetime with respect to the case without exozodiacal disc is more important for larger aperture telescopes. For instance, the tolerable densities are 50, 30 and 15 zodis respectively for 1-m, 2-m and 4-m apertures telescopes in order to survey at least $90 \%$ of the nominal targets. These values become 200,100 and 60 zodis if $75 \%$ of the targets have to be surveyed. Considering again that 150 targets have to be observed during the mission lifetime, dust densities as high as 100 and 600 zodis can be tolerated around the target stars for 2-m and 4-m apertures telescopes respectively (150 targets being not detectable within the survey time with $1-\mathrm{m}$ aperture telescopes). Due to the better nominal performance achieved with 4-m aperture telescopes (about 500 targets surveyed during the survey time, see Table 4), the maximum dust density to survey 150 targets (600 zodis) is higher than for 2-m aperture telescopes (100 zodis) but the corresponding loss of surveyed targets with respect to the nominal case is much more important $\left(R_{\mathrm{obs}}\right.$ of $30 \%$ vs. $\left.75 \%\right)$. In practice, larger aperture telescopes would obviously be better to maximize the scientific performance but the final choice will also result from a trade-off with cost and feasibility.

\section{Impact of the exozodiacal cloud morphology}

The previous results have been obtained assuming that the exozodiacal dust emission is centrally symmetric around the target star so that it is suppressed by phase chopping (and therefore only contributes to shot noise). However, exozodiacal discs are likely to show resonant structures or an offset with respect to the central star due to the gravitational influence of embedded planets. These resonant structures have been predicted by theoretical studies (Roques et al. 1994; Liou \& Zook 1999; Ozernoy et al. 2000), and observed in few cases around nearby main-sequence stars (e.g., Wilner et al. 2002; Greaves et al. 2005; Kalas et al. 2005; Schneider et al. 2009). The most well-studied example of an asymmetric disc is the solar zodiacal cloud, which exhibits several structures interpreted as the dynamical signature of planets (Dermott et al. 1985, 1994; Reach et al. 1995). This trend suggests that exozodiacal clouds may be full of rings, clumps, and other asymmetries induced by the presence of embedded planets. These asymmetric structures around the target star are not perfectly canceled by the phase chopping process and part of the exozodi signal can then mimic the planetary signal. If the demodulated contribution from the exozodiacal disc is significantly higher than that of the planet, it would be difficult to isolate the planetary signal, whatever the integration time. As mentioned by Lay (2004), asymmetric inhomogeneities at the $0.1 \%$ level of the total exozodiacal flux can be confused with a planetary signal. The problem becomes even more serious as the dust density increases: a 10-zodi exozodiacal disc must be smooth at the $0.01 \%$ level, in the region to be searched for planets. Considering asymmetric exozodiacal discs, we derive in this section the tolerable dust density in order to ensure the detection of Earth-like planets with DARWIN/TPF.

\subsection{Methodology}

At the output of the interferometer, the total detected photon rate (excluding stray light) can be written as (Lay 2004):

$N=\int_{\theta} \int_{\alpha} B_{\text {sky }}(s) R(s) P(s) \theta \mathrm{d} \theta \mathrm{d} \alpha$,

where $B_{\text {sky }}$ is the brightness distribution on the sky for a spectral channel centered on wavelength $\lambda$ and a bandwidth $\Delta \lambda \ll \lambda$ (units in photons $/ \mathrm{s} / \mathrm{m}^{2}$ ), $s$ is a unit vector whose direction represents position on the sky, $P(s)$ is a field-of-view taper function resulting from the size of a collecting aperture and from the response of the single-mode spatial filter, and $R(s)$ is the intensity response of the interferometer on the sky (excluding the taper), the so-called transmission map. Equation (1) can be written as a function of the double Fourier transform of the sky brightness, including the field-of-view taper, which are denoted by a horizontal bar:

$$
\begin{aligned}
N= & \sum_{j} \sum_{k} A_{j} A_{k}\left[\cos \left(\phi_{j}-\phi_{k}\right) \bar{B}_{\mathrm{sky}, j k, \mathrm{sym}}\right. \\
& \left.-\sin \left(\phi_{j}-\phi_{k}\right) \bar{B}_{\mathrm{sky}, j k, \text { asym }}\right]
\end{aligned}
$$

where $B_{\text {sky,sym }}$ (resp. $B_{\text {sky,asym }}$ ) is the symmetric (resp. asymmetric) part of the sky brightness distribution and $\phi_{j}$ the phase response of the telescope $j$. The total photon rate is therefore a sum over all possible pairs of collectors. The baselines with a phase difference that is an integer multiple of $\pi(0, \pm \pi, \pm 2 \pi)$ couple entirely to the symmetric brightness distribution (star, local zodiacal cloud, exozodiacal disc). Any asymmetric brightness distribution couples entirely to baselines with a phase difference that is an odd multiple of $\pi / 2$ (e.g., planet, clump). From Eq. (2), the detected photon rate from the exozodiacal disc is simply given by:

$$
\begin{aligned}
N_{\mathrm{EZ}} & =\sum_{j} \sum_{k} A_{j} A_{k}\left[\cos \left(\phi_{j}-\phi_{k}\right) \bar{B}_{\mathrm{EZ}, j k, \mathrm{sym}}\right. \\
& \left.-\sin \left(\phi_{j}-\phi_{k}\right) \bar{B}_{\mathrm{EZ}, j k, \text { asym }}\right]
\end{aligned}
$$

The demodulated signal from the exozodiacal disc can then be obtained (after combination of the two chop states):

$O_{\mathrm{EZ}}=\frac{1}{T} \int_{0}^{T}\left(N_{\mathrm{EZ}}^{L}-N_{\mathrm{EZ}}^{R}\right) \eta \mathrm{d} t$

where $T$ is the integration time, $N_{\mathrm{EZ}}^{L}\left(\right.$ resp. $\left.N_{\mathrm{EZ}}^{R}\right)$ the contribution of the exozodiacal disc at the output of the left (resp. right) chop state and $\eta$ the demodulation template function. This template function is used to extract the planetary signal by crosscorrelation (Angel \& Woolf 1997). It represents the time series (normalized to have a rms value of one) that would be obtained, should a planet be present at a given position. Taking into account that the phase response of the interferometer is such that $\phi_{i}^{L}=-\phi_{i}^{R}$ with the $\phi_{i}^{L}$ equal to $(\pi / 2,0, \pi, 3 \pi / 2)$, Eqs. (3) and (4) give:

$O_{\mathrm{EZ}}=\frac{2}{T} \sum_{j} \sum_{k} A_{j} A_{k} \sin \left(\phi_{j}-\phi_{k}\right) \int_{0}^{T} \bar{B}_{\mathrm{EZ}, j k, \mathrm{asym}} \eta \mathrm{d} t$,

which shows that only asymmetric components of the brightness distribution contribute to the demodulated signal. More specifically, only the baselines with a "fractional- $\pi$ " phase difference can produce a demodulated signal. Such baselines are mandatory in order to generate odd harmonics of the rotation frequency. A 

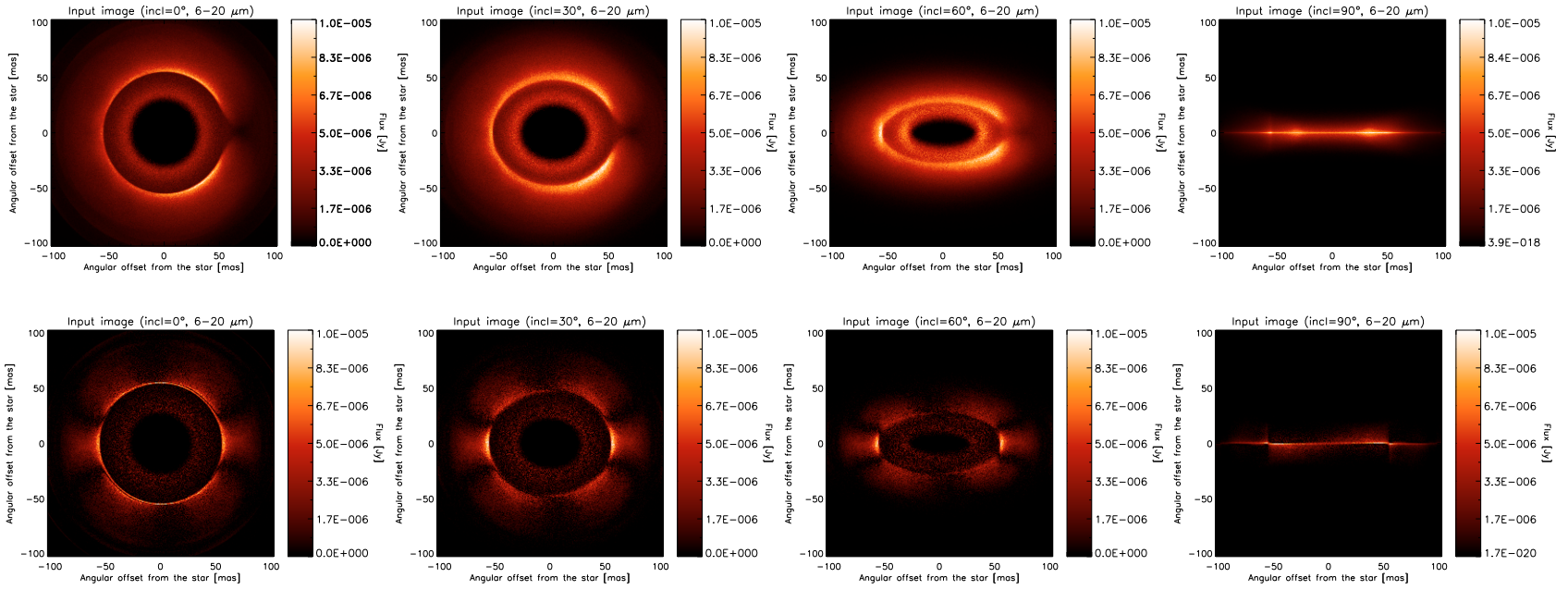

Fig. 11. Upper: thermal flux $(6-20 \mu \mathrm{m})$ produced by a 10-zodi exozodiacal dust cloud around a G2V star for four different disc inclinations $\left(0^{\circ}\right.$, $30^{\circ}, 60^{\circ}$ and $90^{\circ}$ ) and assuming a Dohnanyi distribution of particle sizes (Dohnanyi 1969). The images have been simulated assuming a Earth-mass planet located at $1 \mathrm{AU}$ on the $x$-axis (90 degrees clockwise from vertical, Stark \& Kuchner 2008). Lower: corresponding asymmetric brightness distributions obtained by subtracting to each pixel its centrally symmetric counterpart.

solution to mitigate the influence of the asymmetric structures in the disc is to have long imaging ("fractional- $\pi$ ") baselines that resolve out the more spatially extended emission from the exozodi variations, leaving only the point like emission from planets.

\subsection{Impact of clumps}

The origin of some asymmetric clumpy structures, i.e. local density enhancements, in exozodiacal discs may be attributed to to the gravitational influence of planets on the small dust grains. After their release from parent bodies via collisions or outgassing, dust grains experience different paths in the stellar system, depending on their effective size. Whereas the smallest particles are ejected from the planetary systems by radiation pressure in a dynamical time, larger particles slowly spiral inward due to Pointing-Robertson drag (Robertson 1937). While spiraling toward their host star, dust particles may become temporarily trapped in mean motion resonance with planets, extending their lifetimes. This trapping locally enhances the particle density, creating structures, originally described for the solar zodiacal cloud as circumstellar rings, bands, and clumps (e.g., Kelsall et al. 1998).

In order to address the impact of such structures on the performance of DARWIN/TPF, we use the results of Stark \& Kuchner (2008), who synthesized images of circumstellar discs with resonant rings structures due to embedded terrestrial-mass planets. Among the studies that have examined the geometry of these resonant signatures (e.g., Kuchner \& Holman 2003; Moro-Martín \& Malhotra 2005; Reche et al. 2008; Stark \& Kuchner 2008), these images are particularly convenient for our study since they include enough particles to overcome the limitations of previous simulations, which were often dominated by various sources of Poisson noise, and allow for quantitative study of the modeled ring structures. In addition, these images are geared toward terrestrial-mass planets at a few AU from the star, whereas most other studies concern more massive planets located much farther from the star. We used the Stark \& Kuchner (2008) models to produce thermal emission images of inclined $\operatorname{discs}\left(0^{\circ}, 30^{\circ}, 60^{\circ}\right.$ and $\left.90^{\circ}\right)$ with resonant ring structures. We investigated disc models for a system with an Earth-mass planet on a circular orbit at $1 \mathrm{AU}$ around a G2V star located at $15 \mathrm{pc}$ and for a Dohnanyi distribution ranging in size from the blowout size up to $120 \mu \mathrm{m}$ (Dohnanyi 1969). The thermal emission produced by such exozodiacal discs are given in the upper part of Fig. 11 in a wavelength range of $6-20 \mu \mathrm{m}$.

The images given in Fig. 11 can be thought of as upper-limits to the brightness of structures due to an Earth-like planet. Stark \& Kuchner (2008) ignored dust from parent bodies with large inclinations and eccentricities, such as comets, which would tend to wash out any resonant structure. Additionally, these models ignore the effects of collisions, which smooth out overdense regions of the disc and reduce azimuthal asymmetries (Stark \& Kuchner 2009). In every simulation, the parent bodies were initially distributed from 3.5 to $4.5 \mathrm{AU}$ in an asteroid belt-like ring. The Earth-mass planet is oriented along the $x$-axis (located at 66 mas on the $x$-axis) with a noticeable gap in the ring at its position. The models are truncated at half the semi-major axis of the planet, resulting in the inner holes in the images of Fig. 11. In reality, the dust density distribution should continue inward to the dust sublimation radius in the absence of additional perturbers. This "missing" inner disc should however not affect our results, since the inner disc would be centrally symmetric so that it does not contribute to the detected signal.

Introducing these images into DarwinSIM, we compute the chopped photon rate from the exozodiacal disc as a function of the array rotation angle. This is represented in the upper part of Fig. 12 for two different disc inclinations $\left(0^{\circ}\right.$ and $\left.60^{\circ}\right)$ at $10 \mu \mathrm{m}$ (left figures) and for broadband detection (6-20 $\mu \mathrm{m}$, right figures). The density of the disc has been scaled up to 10 zodis and the chopped planetary signal represented for comparison (dashed curve). In all cases, it is dominated by the chopped signal from the exozodiacal disc (solid curve) and particularly for high disc inclinations. To disentangle the planetary signal from the disc signal, it is necessary to apply the cross-correlation method to build the so-called dirty map. Applying cross correlation of the measured signal (disc + planet) with templates of the signal expected from a point source at each location on the sky (computed using Eq. (5)), the dirty maps represented in the lower part of Fig. 12 are obtained. This process transforms the rotationally modulated signal into a map of the sky by crosscorrelation, which is equivalent to the Fourier transform used in 
Table 5. Tolerable exozodiacal dust density for different disc inclinations and wavelengths.

\begin{tabular}{ccccc}
\hline \hline Disc incl. & $8 \mu \mathrm{m}$ & $10 \mu \mathrm{m}$ & $16 \mu \mathrm{m}$ & Wide \\
\hline $0^{\circ}$ & 12.2 & 15.3 & 7.0 & 14.0 \\
$30^{\circ}$ & 9.1 & 15.0 & 6.9 & 13.8 \\
$60^{\circ}$ & 6.1 & 6.8 & 2.1 & 3.8 \\
$90^{\circ}$ & 1.1 & 1.4 & 1.0 & 2.6 \\
\hline
\end{tabular}

The detection threshold is taken to be 5 times the rms deviation of the pixel counts within an annulus of width equal to the size of the PSF at half maximum.

standard synthesis imaging. The planet is located at 66 mas on the $x$-axis and presents a demodulated signal of about $0.028 \mathrm{e}^{-} / \mathrm{s}$ at $10 \mu \mathrm{m}$ and about $0.62 \mathrm{e}^{-} / \mathrm{s}$ for broadband detection. The total demodulated signal at the planet position is however lower due to the negative contribution from the exozodiacal disc which presents a hole near the planet. The demodulation of the exozodiacal discs also produces main peaks which are maximum around 30-50 mas from the host star (in agreement with the asymmetric brightness distribution in the initial images, see the lower part of Fig. 11). Fortunately, the high angular resolution provided by the long imaging baseline is sufficient to spatially distinguish these components from the planetary signal and only the contribution from the hole around the planet significantly contributes to the noise level.

In order to ensure the planet detection, we adopt a criterion commonly used in AO imaging (Macintosh et al. 2003; Hinkley et al. 2007; Serabyn 2009). The noise level is taken to be the rms deviation of the pixel counts within an annulus of width equal to the size of the PSF at half maximum. Considering a detection threshold of 5 , the results are given in Table 5 for different wavelengths and disc inclinations. The tolerable disc density ranges between about 1 and 15 zodis, depending on the disc inclination. The detection is particularly difficult for highly inclined discs for which the asymmetric components are more dominant and at long wavelengths where the planetary signal is weaker. Combining the spectral channels to obtain a broadband correlation map (see Fig. 12) reduces the impact of sidelobes associated with each main peak but does not significantly improve the results. This is because the performance are limited by the main peak induced by the hole near the planet rather than by sidelobes. Note that this hole generally induces a response 2 to 4 times larger the rms deviation of the pixel counts within the annulus so that it might be marginally interpreted as a planet detection (a "false positive"). However, it could also be seen as an indirect way to detect the presence of a planet within the hole since such compact structure is expected to be created by a planet.

In order to relax these stringent constraints on the exozodiacal dust density, more sophisticated techniques of data processing could be useful (e.g., Thiebaut \& Mugnier 2006). The capability of these techniques still needs to be investigated and is beyond the scope of this paper. The only secure way to ensure the detection and characterization of Earth-like planets with DARWIN/TPF-like missions is to observe in advance the nearby main-sequence stars in order to remove from the target list the stars with a too high inclined/bright exozodiacal disc. To achieve this goal, a space-based nulling interferometer such as FKSI ("Fourier-Kelvin Stellar Interferometer") would be ideal with a sensitivity sufficient to detect exozodiacal discs down to 1 zodi (Defrère et al. 2008a).

\subsection{Impact of the disc offset}

An offset between the center of symmetry of a dust cloud and its host star is a natural consequence of the gravitational interaction with planets. In the solar system, the center of the zodiacal cloud is shifted by about $0.013 \mathrm{AU}$ from the Sun due mostly to Jupiter (Landgraf \& Jehn 2001). The offset can be much larger, as shown in the case of the Fomalhaut system with an offset of $15 \mathrm{AU}$ (Kalas et al. 2005). Even when inhomogeneities such as clumps are not present, an offset cloud produces an asymmetric brightness distribution such that a part of the exozodiacal disc signal survives the chopping process. Using the zodipic package ${ }^{4}$, we produce images of solar-like zodiacal discs with a given offset and use them to compute the demodulated signal at the output of the interferometer. The results are presented in Fig. 13, showing the tolerable dust density with respect to the disc offset for a G2V star located at $15 \mathrm{pc}$ and for different wavelengths. The disc is assumed to be seen face-on.

As the distance between the host star and the center of symmetry of the exozodiacal disc increases, the tolerable dust density to detect an Earth-like planet located at 1 AU becomes more severe and reaches less than 5 zodis for an offset of 1 AU. For individual spectral channels, the tolerable dust density rapidly decreases to reach the value of 20 zodis at $0.05,0.15$ and $0.25 \mathrm{AU}$ respectively at $8 \mu \mathrm{m}, 10 \mu \mathrm{m}$ and $16 \mu \mathrm{m}$. The results for broadband detection are much better with a tolerable dust density of 20 zodis only for a disc presenting an offset larger than $0.6 \mathrm{AU}$. Considering a tolerable exozodiacal dust density of 100 zodis, the offset between the host star and the center of symmetry of the exozodiacal disc can be as high as $0.4 \mathrm{AU}$ in order to ensure the planet detection. For an offset similar to that of the solar zodiacal cloud (about $0.013 \mathrm{AU}$ ), this tolerable dust density is even much higher (few thousand zodis).

\section{Conclusions}

Infrared nulling interferometry is the core technique of future life-finding space missions such as ESA's DARWIN and NASA's Terrestrial Planet Finder (TPF). Observing in the infrared $(6-20 \mu \mathrm{m})$, these missions will be able to characterise the atmosphere of habitable extrasolar planets orbiting around nearby main sequence stars. This ability to study distant planets strongly depends on exozodiacal clouds around the stars, which can hamper the planet detection. Considering the nominal mission architecture with 2-m aperture telescopes, we show that centrally symmetric exozodiacal dust discs about 100 times denser than the solar zodiacal cloud can be tolerated in order to survey at least 150 targets during the mission lifetime. The actual number of planet detections will then depend on the number of terrestrial planets in the habitable zone of target systems.

The presence of asymmetric structures in exozodiacal discs (e.g., clumps or offset) may be more problematic. While the cloud brightness drives the integration time necessary to disentangle the planetary photons from the background noise, the emission from inhomogeneities are not perfectly subtracted by phase chopping so that a part of the disc signal can mimic the planet. To address this issue, we consider modeled resonant structures produced by an Earth-like planet and introduce the corresponding image into DarwinSIM, the mission science simulator. Even for exozodiacal discs a few times brighter than the solar zodiacal cloud, the contribution of these asymmetric structures can be much larger than the planetary signal at the output

\footnotetext{
${ }^{4}$ http//asd.gsfc.nasa.gov/Marc.Kuchner/home.html
} 

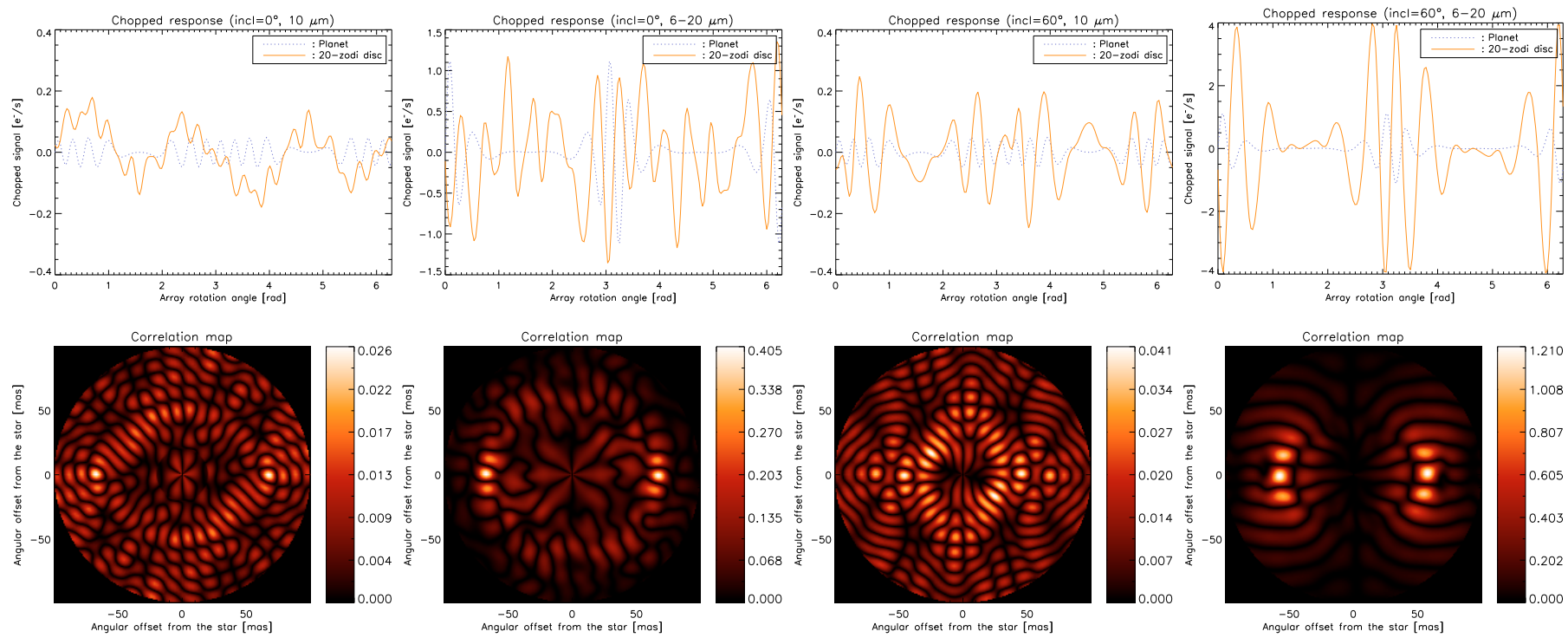

Fig. 12. Upper: chopped photon rate from an Earth-like planet and a 10-zodi asymmetric disc with respect to the rotation angle for two disc inclinations $\left(0^{\circ}\right.$ and $\left.60^{\circ}\right)$ and different wavelength ranges (at $10 \mu \mathrm{m}$ and in the full wavelength range). Lower: corresponding dirty map formed from the cross correlation of the measured signal with templates of the signal expected from a point source at each location on the sky.

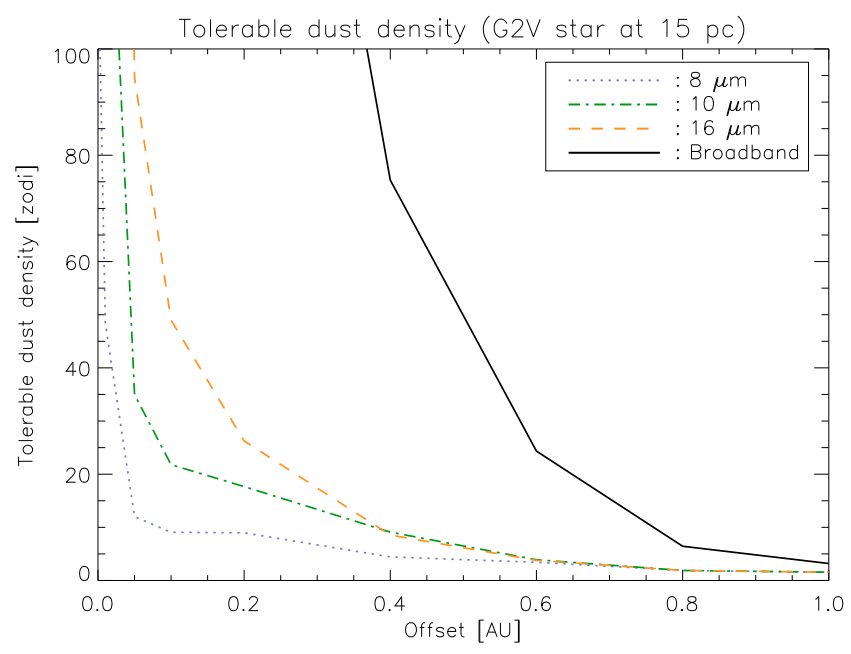

Fig. 13. Tolerable exozodiacal dust density with respect to the offset between the center of symmetry of the exozodiacal disc and the central star (a G2V star located at $15 \mathrm{pc}$ ). The disc is assumed to be seen in face-on orientation.

of the interferometer. Fortunately, the high angular resolution provided by the long imaging baseline of DARWIN/TPF in the $\mathrm{X}$-array configuration is sufficient to spatially distinguish most of the extended exozodi emission from the planetary signal and only the hole in the dust distribution near the planet significantly contributes to the noise level. Considering the full wavelength range of DARWIN/TPF, we show that the tolerable dust density is about 15 times the solar zodiacal density for face-on systems and decreases with the disc inclination. In practice, this constraint might be relaxed since we examined a resonant ring model that does not include dust from highly eccentric or inclined parent bodies, the effects of grain-grain collisions, or perturbations by additional planets, all of which can reduce the contrast of the resonant ring and improve the tolerance to the exozodiacal dust density.

These results show that asymmetric structures in exozodiacal discs around nearby main sequence stars are one of the main noise sources for future exo-Earth characterization missions. A first solution to get around this issue is to have a long imaging baseline architecture which resolves out the more spatially extended emission of the exozodiacal cloud from the point-like emission of planets. The stretched X-array configuration is particularly convenient in that respect. The second solution is to observe in advance the nearby main sequence stars and remove from the DARWIN/TPF target list those presenting a too high dust density or disc inclination. The FKSI nulling interferometer would be ideal in that respect with the possibility to detect exozodiacal discs down to the density of the solar zodiacal cloud. Ground-based nulling instruments like LBTI and ALADDIN would also be particularly valuable.

Acknowledgements. The authors are grateful to Lisa Kaltenegger (HarvardSmithsonian Center for Astrophysics) for providing the updated DARWIN catalogue, Jean Surdej (IAGL), Arnaud Magette (IAGL), Dimitri Mawet (NASA/JPL), Peter Lawson (NASA/JPL), Oliver Lay (NASA/JPL), Pierre Riaud (IAGL) and Virginie Chantry (IAGL). This research was supported by the International Space Science Institute (ISSI) in Bern, Switzerland ("Exozodiacal Dust discs and Darwin" working group, http://www.issibern.ch/teams/ exodust/). D.D. and C.H. acknowledge the support of the Belgian National Science Foundation ("FRIA"). O.A. acknowledges the support from a F.R.S.FNRS Postdoctoral Fellowship. D.D. and O.A. acknowledge support from the Communauté française de Belgique - Actions de recherche concertées Académie universitaire Wallonie-Europe.

\section{Appendix A: Deriving instability noise constraints}

We address here the instability noise and derive the constraints on the instrument stability required to detect an Earth-like planet orbiting at $1 \mathrm{AU}$ around a G2V star located at $15 \mathrm{pc}$. The analysis follows the analytical method of Lay (2004) which was originally applied to the DCB configuration at $10 \mu \mathrm{m}$. The goal is to extend the study to the X-array architecture and to short wavelengths where instability noise is the most dominant. We define the limiting rms OPD and amplitude errors such that instability noise is dominated by a factor 5 by shot noise over a single rotation of $50000 \mathrm{~s}$. Assuming that instability noise is totally uncorrelated with the rotation angle, this factor stays unchanged over multiple rotations. In practice, instability noise can be correlated with the rotation angle due to perturbations such as solar heating 
D. Defrère et al.: Impact of exozodiacal clouds on the performance of future life-finding space missions

Table A.1. Limiting rms OPD values computed for a G2V star located at 15 pc such that shot noise dominates instability noise by a factor 5 .

\begin{tabular}{|c|c|c|c|c|c|c|c|c|c|c|c|c|c|}
\hline \multirow[t]{3}{*}{$\sigma_{\mathrm{OPD}}[\mathrm{nm}]$} & \multirow[b]{3}{*}{$\sigma_{\mathrm{amp}}$} & \multicolumn{4}{|c|}{ 2-m apertures } & \multicolumn{8}{|c|}{ 4-m apertures } \\
\hline & & \multicolumn{3}{|c|}{$\lambda=7 \mu \mathrm{m}$} & \multicolumn{2}{|l|}{$\lambda=10 \mu \mathrm{m}$} & & \multicolumn{3}{|c|}{$\lambda=7 \mu \mathrm{m}$} & \multicolumn{3}{|c|}{$\lambda=10 \mu \mathrm{m}$} \\
\hline & & DCB & $\mathrm{X}-\mathrm{ar} 2$ & X-ar6 & DCB & $\mathrm{X}$-ar2 & X-ar6 & DCB & $\mathrm{X}-\mathrm{ar} 2$ & $\mathrm{X}$-ar6 & $\mathrm{DCB}$ & $\mathrm{X}-\mathrm{ar} 2$ & $\mathrm{X}$-ar6 \\
\hline \multirow[t]{3}{*}{ White noise ${ }^{a}$} & $0.01 \%$ & $>50$ & $>50$ & $>50$ & $>50$ & $>50$ & $>50$ & $>50$ & $>50$ & $>50$ & $>50$ & $>50$ & $>50$ \\
\hline & $0.05 \%$ & 16 & 9.5 & 9.5 & $>50$ & $>50$ & $>50$ & 6.5 & 4.6 & 4.6 & 23 & 16 & 16 \\
\hline & $0.10 \%$ & 2.4 & 1.0 & 1.0 & 5.3 & 4.8 & 4.8 & 0.8 & 0.5 & 0.5 & 3.7 & 2.9 & 2.9 \\
\hline \multirow[t]{3}{*}{$1 /$ f noise $^{b}$} & $0.01 \%$ & 0.7 & 2.6 & 4.2 & 2.8 & 13 & 19 & 0.4 & 1.1 & 1.9 & 1.1 & 3.6 & 5.6 \\
\hline & $0.05 \%$ & 0.7 & 0.9 & 1.5 & 2.0 & 2.9 & 4.1 & 0.3 & 0.4 & 0.6 & 0.8 & 0.9 & 1.4 \\
\hline & $0.10 \%$ & 0.5 & 0.5 & 0.7 & 1.5 & 1.6 & 2.0 & 0.3 & 0.3 & 0.3 & 0.5 & 0.5 & 0.6 \\
\hline
\end{tabular}

${ }^{a} \mathrm{rms}$ values is defined on the $[0-10 \mathrm{~Hz}]$ wavelength range; ${ }^{b} \mathrm{rms}$ values is defined on the $\left[1 / t_{\mathrm{rot}}-10^{4} \mathrm{~Hz}\right]$ wavelength range. The results are given at $7 \mu \mathrm{m}$ and $10 \mu \mathrm{m}$, assuming a spectral resolution of 20 , for different rms amplitude mismatches and shapes of the instability noise power spectrum. Three configurations are shown, the X-array with an aspect ratio of 2 (X-ar2), the X-array with an aspect ratio of 6 (X-ar6) and the Dual Chopped Bracewell (DCB, configuration used in Lay 2004).

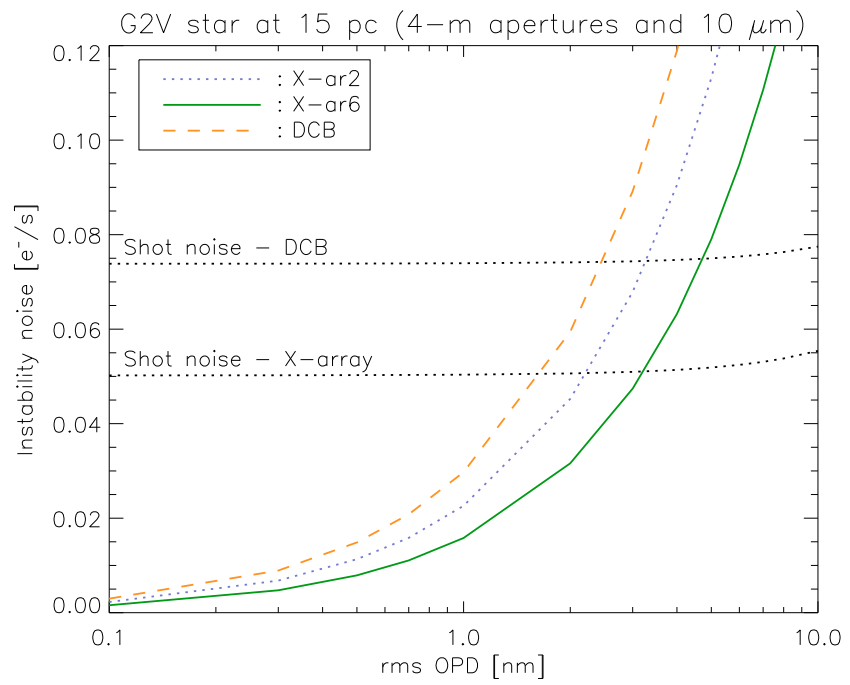

Fig. A.1. Instability noise with respect to the rms OPD errors for different array architectures assuming 1/f-type PSDs and a rms amplitude mismatch of $0.1 \%$ (defined on the $0-10^{4} \mathrm{~Hz}$ frequency range). The level of shot noise is represented by dotted curves for each configuration. The figure has been plotted for 4-m aperture telescopes operating at $10 \mu \mathrm{m}$ and a G2V star located at $15 \mathrm{pc}$ (surrounded by an exozodiacal cloud of 1 zodi).

effects but it should be possible to remove them by measuring and correcting the amplitudes and phases at intervals during the rotation.

For sake of comparison with Lay (2004), we consider first 4-m aperture telescopes operating at $10 \mu \mathrm{m}$ and extend the results to 2-m aperture telescopes operating at $7 \mu \mathrm{m}$ in Table A.1. Assuming a spectral resolution of 20, Fig. A.1 shows instability noise with respect to the rms OPD error for three different configurations: the DCB as defined in Lay (2004), the X-array with a $1: 2$ aspect ratio $(X$-ar2) and the $X$-array with a 1:6 aspect ratio (X-ar6). These curves have been computed assuming 1/f-type PSDs for amplitude and OPD errors with rms values defined on a frequency range from $1 / t_{\text {rot }}$ to $10^{4} \mathrm{~Hz}$, where $t_{\text {rot }}$ is the rotation period, and a G2V star located at $15 \mathrm{pc}$. Shot noise is represented by dotted curves. It is higher for the DCB due to geometrical leakage (the nulling baseline is twice larger than for the X-array) and presents a slight increase for large rms OPD errors due to instrumental leakage. Instability noise is also higher for the DCB than for the X-array. This is because the planetary signal is mostly modulated at lower frequencies where the
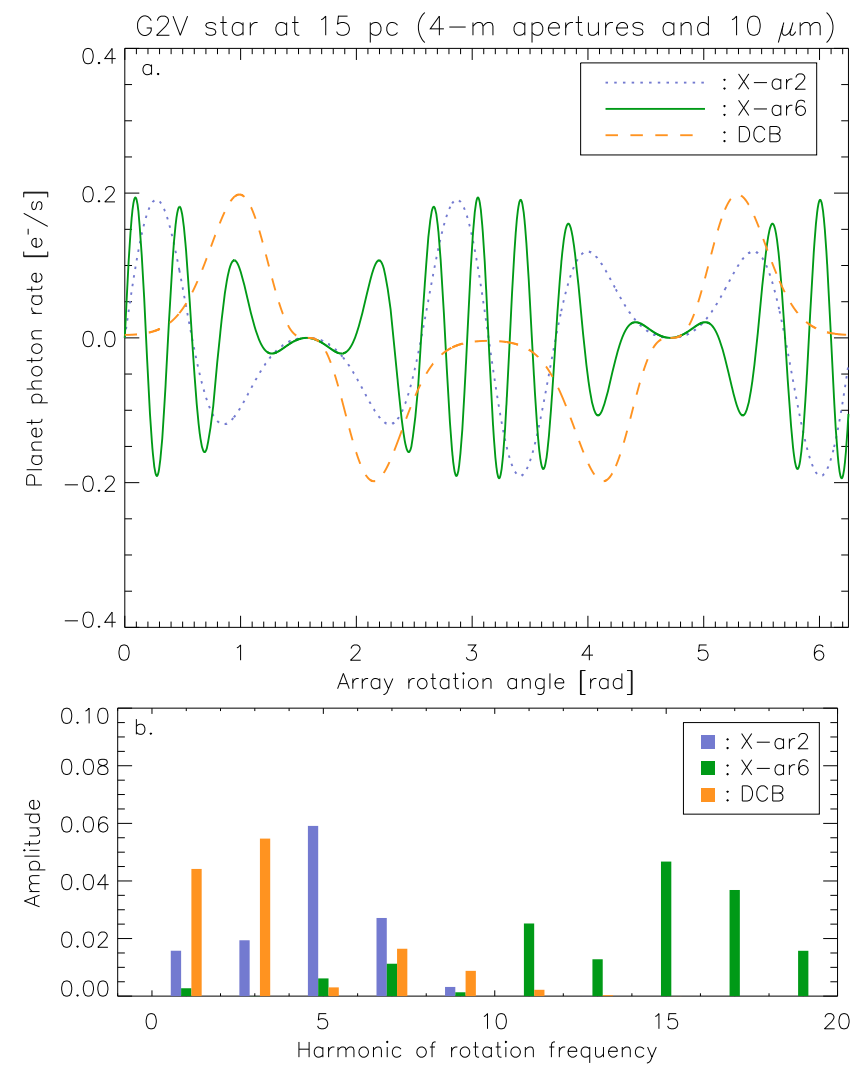

Fig. A.2. Upper: chopped planet detected photon rate as a function of array rotation angle for the different architectures. The planet is assumed to be located at 47 mas from a G2V star located at 15 pc. Lower: corresponding Fourier amplitudes. Only odd harmonics are present because of phase chopping.

instability noise is higher for 1/f-type noises. This is illustrated in Fig. A.2 showing the chopped planet detection rate with respect to the rotation angle of the array (upper figure) and the corresponding Fourier amplitudes (lower figure).

Figure A. 1 shows that shot noise dominates instability noise by a factor 5 for rms OPD errors of about $0.5 \mathrm{~nm}, 0.5 \mathrm{~nm}$ and $0.6 \mathrm{~nm}$ respectively for the DCB, X-ar2 and X-ar6. The slight discrepancy that can be mentioned with Lay (2004) is due to two factors. In addition to the instrument throughput of $10 \%$ used in Lay (2004), our study accounts for the coupling efficiency (about $72 \%$ for the on-axis light) and for the quantum efficiency of the 
detectors $(70 \%)$. We also combine the two chop states whereas the results of Lay (2004) are given for only one. Although instability noise is higher for the DCB configuration than for the $\mathrm{X}$-array, the limiting rms opd error is of the same order due to the higher shot noise. Because instability noise is directly proportional to the stellar flux, the constraints are even more stringent at $7-\mu \mathrm{m}$ where the star is brighter and the level of shot noise basically the same than at $10 \mu \mathrm{m}$ (see Table A.1). Considering an rms amplitude mismatch of $0.1 \%$, the OPD has to be controlled to a level of $0.3 \mathrm{~nm}$ rms for the three considered configurations. These constraints are slightly relaxed for 2-m aperture telescopes because shot noise is relatively more dominant (shot noise is proportional to the squared root of the stellar flux while instability is directly proportional to the stellar flux). For the results at $7 \mu \mathrm{m}$, we use the same baseline length of $20 \mathrm{~m}$ with the same rotation period of $50000 \mathrm{~s}$. These requirements are very stringent and are only marginally compliant with state-of-the-art active control, so that potential ways to mitigate the harmful effect of instability noise have been investigated.

- A first solution, proposed by Lay (2006), consists in stretching the array and applying a low-order polynomial fit to the instability noise (as a function of wavelength). By stretching the array, i.e. increasing the imaging baseline of the $\mathrm{X}$-array, the interference pattern orthogonal to the nulling pattern shrinks. As the modulation map scales with wavelength, the planetary signal transmitted by the interferometer will then be a rapidly oscillation function of wavelength. On the other hand, instability noise is shown to be a loworder polynomial of the optical frequency $(1 / \lambda)$. Therefore, by removing a low-order polynomial fit to the detected signal as a function of wavelength, the instability noise contribution is efficiently subtracted while preserving most of the planetary signal. This operation can actually be performed directly in the cross-correlation, by using a modified planet template where the polynomial components have been removed. Because this method strongly relies on the separation of the nulling and imaging baselines, it can only be efficiently applied with the X-array architecture.

- Another solution, based on the coherence properties of starlight, has been proposed by Lane et al. (2006) to separate the contributions from the planet and the instrumental leakage. The idea is to mix the electric fields of the leakage with that of a separate reference beam, also from the star, in order to form fringes (as long as the relative path delays are maintained within the coherence length). The light from the planet, being not coherent with the starlight, will not form fringes. Using as reference beam the bright output of a pairwise nulling beam combiner, the amplitude and phase mismatches in the input beams can be extracted from the fringe pattern, allowing the reconstruction of the the stellar leakage.

- A third solution has recently been proposed and tested at IAS (Gabor et al. 2008). The principle is to successively apply two $(<\lambda / 2)$ opposite OPD offsets to one of the beams in order to derive the actual position of the minimum in the transmission map. In this way, one feeds back the actual OPD errors to the delay line and prevents drifts from appearing. The same principle can be used to avoid amplitude drifts, by either blocking all but one beam to measure its actual amplitude or by modulating its amplitude as in the case of the OPD. The frequency at which this process is carried out depends on the input power spectra of the noise sources. It has been demonstrated experimentally in the lab that this process efficiently suppresses the 1/f-type noise generally present in the stellar contribution at the output of a Bracewell interferometer. This technique can theoretically be applied to any nulling configuration, but its efficiency decreases as the number of beams increases.

Others possible approaches (e.g. application of closure phase techniques, new chopping processes, exploiting correlations between measurements taken at different wavelength bins) have been suggested but still need to be investigated. However, these very stringent requirements have been derived with the assumption that the instability noise present an 1/f-type power spectrum. In fact, the shape of the instability noise spectrum strongly depends on the input spectrum of noise fluctuations. Pure 1/f noise might in fact turn out to be a very pessimistic assumption. Given that the DARWIN/TPF system is designed to have three levels of control loops that manage the OPD, and two control loop levels for tip/tilt, the resulting residual phase and intensity fluctuations that are responsible for the instability noise are most likely to have a flat input spectrum. This has a strong influence on the magnitude of the phase-amplitude cross terms, which are the main contributor to instability noise. For instance, the tolerable rms OPD errors for a white spectrum defined on the $[0-10 \mathrm{~Hz}]$ frequency range are significantly relaxed as indicated in Table A.1. In particular at low frequencies, where the planetary signal is modulated and the instability noise arises, the 1/f spectrum diverges, while the flat spectrum increases linearly. In practice, the situation might even be better, as predictive or Kalman filtering may be used to remove low-frequency power. A complication, in particular for the micro $\mathrm{Hz}$ domain, is that zero point drifts of the control loops, e.g. related to electronic drifts in the sensors, remain undetected, and thus uncorrected. They will result in a low-frequency $1 / \mathrm{f}$ component. A simple solution is to switch the incoming beams with respect to the control loops. The reason why this could work is that the extremely high precision required for DARWIN/TPF is only on relative quantities, namely, the phase and amplitude differences between any two beams. Absolute offsets of phase and amplitude, that apply to all three beams simultaneously, do not contribute to the instability noise, since they do not affect the null. The beams must be switched with respect to the control inputs with a time constant over which the drift can be considered constant. In summary, even though at present a post-processing method has not been identified, it is highly likely that clever and careful engineering may already reduce instability noise to harmless levels.

\section{References}

Absil, O. 2001, Master's thesis, Liège University, Liège, Belgium, http://www . aeos.ulg.ac.be/

Absil, O. 2006, Ph.D. Thesis, Liège University, Liège, Belgium Absil, O., di Folco, E., Mérand, A., et al. 2006, A\&A, 452, 237 Absil, O., Defrère, D., Coudé du Foresto, V., et al. 2008a, in Proc. SPIE, 7013 Absil, O., di Folco, E., Mérand, A., et al. 2008b, A\&A, 487, 1041 Absil, O., Mennesson, B., Le Bouquin, J.-B., et al. 2009, ApJ, 704, 150 Akeson, R. L., Ciardi, D. R., Millan-Gabet, R., et al. 2009, ApJ, 691, 1896 Alonso, R., Auvergne, M., Baglin, A., et al. 2008, A\&A, 482 Angel, J. R. P., \& Woolf, N. J. 1997, ApJ, 475, 373

Barge, P., Baglin, A., Auvergne, M., et al. 2008, A\&A, 482

Beichman, C. A., Bryden, G., Stapelfeldt, K. R., et al. 2006, ApJ, 652, 1674

Borucki, W. J., Koch, D. G., Lissauer, J., et al. 2007, in Transiting Extrapolar Planets Workshop, ed. C. Afonso, D. Weldrake, \& T. Henning, ASP Conf. Ser., 366, 309

Bracewell, R. N. 1978, Nature, 274, 780

Carle, E. 2005, EMMA configuration: evaluation of optical performances, Tech. Rep. Issue 1, ESA (SCI-A/279)

Chazelas, B., Brachet, F., Bordé, P., et al. 2006, Appl. Opt., 45, 984

D'Arcio, L. 2005, Darwin Variability noise sensitivity analysis, Tech. Rep. Issue 1, ESA (SCI-A/048) 
D. Defrère et al.: Impact of exozodiacal clouds on the performance of future life-finding space missions

Defrère, D., Absil, O., Coudé Du Foresto, V., Danchi, W. C., \& den Hartog, R. 2008a, A\&A, 490, 435

Defrère, D., Lay, O., den Hartog, R., et al. 2008b, in Proc. SPIE, 7013

den Hartog, R. 2005a, DARWIN science performance prediction, Tech. Rep. Issue 1, ESA (SCI-A/300)

den Hartog, R. 2005b, The DARWINsim science simulator, Tech. Rep. Issue 1, ESA (SCI-A/297)

Dermott, S. F., Nicholson, P. D., Burns, J. A., et al. 1985, in Properties and Interactions of Interplanetary Dust, ed. R. H. Giese, \& P. Lamy, Ap\&SS Library, 119, IAU Colloq. 85, 395

Dermott, S. F., Jayaraman, S., Xu, Y. L., Gustafson, B. A. S., \& Liou, J. C. 1994, in , 719

Di Folco, E., Absil, O., Augereau, J.-C., et al. 2007, A\&A, 475, 243

Dohnanyi, J. S. 1969, J. Geophys. Res., 74, 2531

Fridlund, M. 2005, Darwin Science Requirements Document, Tech. Rep. Issue 5, ESA (SCI-A)

Fridlund, C. V. M., d'Arcio, L., den Hartog, R., et al. 2006, in Proc. SPIE, 6268

Gabor, P., Chazelas, B., Brachet, F., et al. 2008, A\&A, 483

Gillon, M., Pont, F., Demory, B.-O., et al. 2007, A\&A, 472, L13

Greaves, J. S., Holland, W. S., Wyatt, M. C., et al. 2005, ApJ, 619, L187

Hinkley, S., Oppenheimer, B. R., Soummer, R., et al. 2007, ApJ, 654, 633

Hinz, P. M., Bippert-Plymate, T., Breuninger, A., et al. 2008, in Proc. SPIE, 7013

Kalas, P., Graham, J. R., \& Clampin, M. 2005, Nature, 435, 1067

Kaltenegger, L., Eiroa, C., \& Fridlund, C. V. M. 2008, A\&SS, accepted [arXiv:0810.5138]

Karlsson, A. L., Wallner, O., Perdigues Armengol, J. M., et al. 2004, in Proc. SPIE, ed. W. A. Traub, 5491, 831

Kelsall, T., Weiland, J. L., Franz, B. A., et al. 1998, ApJ, 508, 44

Ksendzov, A., Lay, O., Martin, S., et al. 2007, Appl. Opt., 46, 7957

Kuchner, M. J., \& Holman, M. J. 2003, ApJ, 588, 1110

Landgraf, M., \& Jehn, R. 2001, Ap\&SS, 278, 357

Lane, B. F., Muterspaugh, M. W., \& Shao, M. 2006, ApJ, 648, 1276

Lawson, P. R., Lay, O. P., Martin, S. R., et al. 2008, in Proc. SPIE, 7013
Lay, O. P. 2004, Appl. Opt., 43, 6100

Lay, O. P. 2005, Appl. Opt., 44, 5859

Lay, O. P. 2006, in Proc. SPIE, 6268

Lay, O. P., Martin, S. R., \& L., H. S. 2007, in Proc. SPIE, 6693

Léger, A., \& Herbst, T. 2007, ArXiv e-prints, 707

Léger, A., Rouan, D., Schneider, J., et al. 2009, A\&A, 506, 287

Liou, J.-C., \& Zook, H. A. 1999, AJ, 118, 580

Macintosh, B. A., Becklin, E. E., Kaisler, D., Konopacky, Q., \& Zuckerman, B. 2003, ApJ, 594, 538

Mawet, D., Hanot, C., Lenaers, C., et al. 2007, Optics Express, 15, 12850

Mayor, M., \& Queloz, D. 1995, Nature, 378, 355

Mayor, M., Bonfils, X., Forveille, T., et al. 2009, A\&A, 507, 487

Mennesson, B., \& Mariotti, J. M. 1997, Icarus, 128, 202

Moro-Martín, A., \& Malhotra, R. 2005, ApJ, 633, 1150

Moutou, C., Pont, F., Barge, P., et al. 2005, A\&A, 437, 355

Ozernoy, L. M., Gorkavyi, N. N., Mather, J. C., et al. 2000, ApJ, 537, L147

Reach, W. T., Franz, B. A., Weiland, J. L., et al. 1995, Nature, 374, 521

Reche, R., Beust, H., Augereau, J.-C., et al. 2008, A\&A, 480, 551

Richardson, L. J., Deming, D., Horning, K., Seager, S., \& Harrington, J. 2007, Nature, 445,892

Robertson, H. P. 1937, MNRAS, 97, 423

Roques, F., Scholl, H., Sicardy, B., et al. 1994, Icarus, 108, 37

Ruilier, C., \& Cassaing, F. 2001, J. Opt. Soc. Amer. A, 18, 143

Schneider, G., Weinberger, A. J., Becklin, E. E., Debes, J. H., \& Smith, B. A. 2009, AJ, 137, 53

Serabyn, E. 2009, ApJ, 697, 1334

Serabyn, E., \& Colavita, M. M. 2001, Appl. Opt., 40, 1668

Stark, C. C., \& Kuchner, M. J. 2008, ApJ, 686, 637

Stark, C. C., \& Kuchner, M. J. 2009, ApJ, 707, 543

Stark, C. C., Kuchner, M. J., Traub, W. A., et al. 2009, BAAS 41, 501

Thiebaut, E., \& Mugnier, L. 2006, in Direct Imaging of Exoplanets: Science and Techniques, IAU Colloq. 200, 547

Wilner, D. J., Holman, M. J., Kuchner, M. J., et al. 2002, ApJ, 569, L115 